\title{
Non-perturbative determination of the $\Lambda$-parameter in the pure SU(3) gauge theory from the twisted gradient flow coupling
}

\author{
Ken-Ichi Ishikawa, ${ }^{a, b}$ Issaku Kanamori, ${ }^{a}$ Yuko Murakami, ${ }^{a}$ Ayaka Nakamura, ${ }^{a}$ \\ Masanori Okawa ${ }^{a, b}$ and Ryoichiro Ueno ${ }^{a}$ \\ ${ }^{a}$ Graduate School of Science, Hiroshima University, \\ Higashi-Hiroshima, Hiroshima 739-8526, Japan \\ ${ }^{b}$ Core of Research for the Energetic Universe, Hiroshima University, \\ Higashi-Hiroshima, Hiroshima 739-8526, Japan \\ E-mail: ishikawa@theo.phys.sci.hiroshima-u.ac.jp, \\ kanamori@hiroshima-u.ac.jp, d152338@hiroshima-u.ac.jp, \\ ayaka@theo.phys.sci.hiroshima-u.ac.jp, okawa@sci.hiroshima-u.ac.jp, \\ ryoichiro-ueno@hiroshima-u.ac.jp
}

Abstract: We evaluate the $\Lambda$-parameter in the $\overline{\mathrm{MS}}$ scheme for the pure $\mathrm{SU}(3)$ gauge theory with the twisted gradient flow (TGF) method. A running coupling constant $g_{\text {TGF }}^{2}(1 / L)$ is defined in a finite volume box with size of $L^{4}$ with the twisted boundary condition. This defines the TGF scheme. Using the step scaling method for the TGF coupling with lattice simulations, we can evaluate the $\Lambda$-parameter non-perturbatively in the TGF scheme. In this paper we determine the dimensionless ratios, $\Lambda_{\mathrm{TGF}} / \sqrt{\sigma}$ and $r_{0} \Lambda_{\mathrm{TGF}}$ together with the $\Lambda$-parameter ratio $\Lambda_{\mathrm{SF}} / \Lambda_{\mathrm{TGF}}$ on the lattices numerically. Combined with the known ratio $\Lambda_{\overline{\mathrm{MS}}} / \Lambda_{\mathrm{SF}}$, we obtain $\Lambda_{\overline{\mathrm{MS}}} / \sqrt{\sigma}=0.5315(81)\left({ }_{-48}^{+269}\right)$ and $r_{0} \Lambda_{\overline{\mathrm{MS}}}=0.6062(92)\left({ }_{-52}^{+309}\right)$, where the first error is statistical one and the second is our estimate of systematic uncertainty.

KEYwords: Lattice Quantum Field Theory, Renormalization Group

ARXIV EPRINT: 1702.06289 


\section{Contents}

1 Introduction 1

2 Twisted gradient flow coupling $\quad 3$

3 Overview of strategy and simulation details 5

$4 \quad$ TGF running coupling constant and $L_{\max } \Lambda_{\text {TGF }} \quad 7$

5 Physical scale in terms of $L_{\max } \quad 11$

$6 \quad \Lambda$-parameter ratio $\Lambda_{\mathrm{SF}} / \Lambda_{\mathrm{TGF}}$ and $\Lambda_{\overline{\mathrm{MS}}} \quad 15$

$\begin{array}{lll}7 & \text { Summary } & 18\end{array}$

$\begin{array}{ll}\text { A Derivation of the action } & 19\end{array}$

B The number of the configurations for $g_{\text {TGF }}^{2} \quad 20$

C Tables to evaluate $L_{\text {max }} / A_{\text {phys }} \quad 22$

\section{Introduction}

The $\Lambda$-parameter is a fundamental quantity in asymptotically free gauge theories and plays the role to set the scale of the theory. $\Lambda$ characterizes the low energy non-perturbative physics and its determination is one of the most important tasks in lattice gauge theory. In the pure Yang-Mills theory, $\Lambda$ is the only free parameter of the theory and is determined from the coupling constant. Its value depends on the renormalization scheme. In the $\overline{\mathrm{MS}}$ scheme, for example, it is defined by

$$
\Lambda_{\overline{\mathrm{MS}}}=\mu\left(b_{0} g_{\overline{\mathrm{MS}}}^{2}(\mu)\right)^{-\frac{b_{1}}{2 b_{0}^{2}}} \exp \left[-\frac{1}{2 b_{0} g_{\overline{\mathrm{MS}}}^{2}(\mu)}\right] \exp \left[-\int_{0}^{g_{\overline{\mathrm{MS}}}(\mu)} \mathrm{d} \xi\left(\frac{1}{\beta(\xi)}+\frac{1}{b_{0} \xi^{3}}-\frac{b_{1}}{b_{0}^{2} \xi}\right)\right]
$$

where $g_{\overline{\mathrm{MS}}}^{2}(\mu)$ is the $\overline{\mathrm{MS}}$ coupling renormalized at the renormalization scale $\mu$, and $\beta(\xi)$ is the beta function in the $\overline{\mathrm{MS}}$ scheme. $b_{0}$ and $b_{1}$ are the first two coefficients of the perturbative beta function, $b_{0}=\frac{11}{3} \frac{N_{\mathrm{C}}}{16 \pi^{2}}$ and $b_{1}=\frac{34}{3}\left(\frac{N_{\mathrm{C}}}{16 \pi^{2}}\right)^{2}$, for the pure $\mathrm{SU}\left(N_{\mathrm{C}}\right)$ gauge theory. Since the $\overline{\mathrm{MS}}$ scheme is only defined perturbatively, the non-perturbative estimate of $\Lambda_{\overline{\mathrm{MS}}}$ thoroughly within the $\overline{\mathrm{MS}}$ scheme is impossible. Therefore we usually convert a $\Lambda$-parameter determined with a non-perturbative scheme to $\Lambda_{\overline{\mathrm{MS}}}$ through the perturbative relation. 
On the lattice, the $\Lambda$-parameter can be defined by

$$
\begin{aligned}
& \Lambda_{\text {Lat }}=\frac{1}{a}\left(b_{0} g_{\text {Lat }}^{2}(1 / a)\right)^{-\frac{b_{1}}{2 b_{0}^{2}}} \exp \left[-\frac{1}{2 b_{0} g_{\text {Lat }}^{2}(1 / a)}\right] \\
& \times \exp \left[-\int_{0}^{g_{\text {Lat }}(1 / a)} \mathrm{d} \xi\left(\frac{1}{\beta(\xi)}+\frac{1}{b_{0} \xi^{3}}-\frac{b_{1}}{b_{0}^{2} \xi}\right)\right],
\end{aligned}
$$

with the lattice spacing $a$. The bare coupling $g_{0}$ can be related to the lattice spacing $a$ non-perturbatively and be used as $g_{\text {Lat }}(1 / a)=g_{0}$ in eq. (1.2). This defines a lattice scheme. It is, however, well known that the scaling is largely violated for the range of $g_{0}^{2}$ accessible with the presently available computational power. In the early stage of the lattice studies, it was common to use an improved coupling such as $g_{\mathrm{E}}^{2}=\frac{8 N_{\mathrm{C}}}{N_{\mathrm{C}}^{2}-1}\left(1-u_{\mathrm{p}}\right)$ or $g_{\mathrm{A}}^{2}=g_{0}^{2} / u_{\mathrm{p}}$ with $u_{\mathrm{p}}$ the observed plaquette value $[1,2]$. They exhibit a better scaling property, nonetheless there are only intuitive arguments of "tad-pole improvement" to explain why they work.

Great progresses for evaluating non-perturbatively running coupling constants have been made with the discovery of the step scaling method [3], where the renormalization scale is introduced by the physical box-size of the target system. In this method, one can calculate the running coupling in a wide range of the scale covering both the hadronic scale, where we make the non-perturbative calculation of physical quantities with lattice techniques, and the high energy scale, where we can estimate the $\Lambda$-parameter neglecting higher order corrections. The most successful non-perturbative scheme for the running coupling constant in QCD is the Schrödinger functional (SF) scheme [4-11], in which a specific Dirichlet boundary condition is imposed on the temporal direction of the box. The advantages of the SF scheme are that it is regularization independent and can be defined non-perturbatively. In addition, the calculation of the $\Lambda$-parameter ratio $\Lambda_{\overline{\mathrm{MS}}} / \Lambda_{\mathrm{SF}}$ has been done in ref. [12] perturbatively. In the SF scheme, on the other hand, it becomes difficult to calculate the coupling at larger physical box sizes (i.e. low energy renormalization scale) due to the appearance of exceptional configurations and the noisy behavior which result the large statistical error [6].

Several other schemes are also available to define the running coupling with the step scaling method [13-17]. The gradient flow scheme is one of the applications of the gradient flow method, in which the gauge field is smeared with the so-called flow equation and the smeared gauge field has a nice perturbative property on the renormalizability [18-20]. In ref. [21], a renormalized coupling via the gradient flow in a finite size box with the periodic boundary condition has been introduced. However, $\Lambda_{\overline{\mathrm{MS}}}$ cannot be extracted from the coupling, since the coupling has a non-analytic expansion in $\alpha_{\overline{\mathrm{MS}}}$ due to the zero-mode of the gauge field in the periodic boundary condition. To avoid the zero-mode problem, the twisted boundary condition has been introduced by Ramos [22]. The renormalized coupling defined in a finite box with the twisted boundary condition (the TGF scheme) has the normal one-loop relation to the $\overline{\mathrm{MS}}$ scheme and is regularization independent. The running can be traced via the step scaling method on the lattice. The TGF running coupling for the pure $\mathrm{SU}(2)$ Yang-Mills theory has been evaluated using the step scaling method [22] and extended to two- or three-color many flavor dynamical simulations [23, 24]. 
The gradient flow coupling with the Schrödinger functional boundary condition is another scheme avoiding the zero-mode problem and has been investigated in refs. [25-27] for the $\mathrm{SU}(3)$ gauge theories.

We extend Ramos's work [22] to the pure SU(3) Yang-Mills theory. In addition to this, we extract the $\Lambda$-parameter in the TGF scheme and convert it to the $\overline{\mathrm{MS}}$ scheme. The ratio $\Lambda_{\overline{\mathrm{MS}}} / \Lambda_{\mathrm{TGF}}$, which is usually evaluated using the perturbation theory, is not yet available at this time (but there is an ongoing study [28]). Since we already know $\Lambda_{\overline{\mathrm{MS}}} / \Lambda_{\mathrm{SF}}$ [12], actually what we have to estimate is the ratio $\Lambda_{\mathrm{SF}} / \Lambda_{\mathrm{TGF}}$. Therefore we estimate $\Lambda_{\mathrm{SF}} / \Lambda_{\mathrm{TGF}}$ for the pure $\mathrm{SU}(3)$ gauge theory numerically with lattice simulations in this study. It should be noted that the analysis made in this paper is applicable to the gauge theories with dynamical fermions provided that the fermion representations and contents are compatible with the twisted boundary condition. This study is the first attempt to apply the TGF method for evaluating the $\Lambda$-parameter in the SU(3) gauge theories from the beginning to the end.

In this study we estimate $\Lambda_{\overline{\mathrm{MS}}}$ in terms of physical observables via the TGF method. Our strategy is summarized as follows:

$$
\frac{\Lambda_{\overline{\mathrm{MS}}}}{A_{\mathrm{phys}}}=\frac{\Lambda_{\overline{\mathrm{MS}}}}{\Lambda_{\mathrm{SF}}} \cdot \frac{\Lambda_{\mathrm{SF}}}{\Lambda_{\mathrm{TGF}}} \cdot \frac{L_{\mathrm{max}} \Lambda_{\mathrm{TGF}}}{L_{\mathrm{max}} A_{\mathrm{phys}}} .
$$

Here $A_{\text {phys }}$ is a physical observable with mass dimension and $L_{\max }$ is an intermediate scale which connects the non-perturbative energy scale and the perturbative energy scale. In this paper, we consider the string tension $\sqrt{\sigma}$ and the Sommer scale $1 / r_{0}$ as the physical observable $A_{\text {phys }}$. (Another reference scale can be considered, for example $w_{0}$ [29].) We will numerically calculate $L_{\mathrm{max}} \Lambda_{\mathrm{TGF}}, L_{\mathrm{max}} A_{\mathrm{phys}}$, and $\Lambda_{\mathrm{SF}} / \Lambda_{\mathrm{TGF}} . L_{\mathrm{max}} \Lambda_{\mathrm{TGF}}$ is calculated with the step scaling method. In order to evaluate $L_{\text {max }} A_{\text {phys }}$, we employ data available from refs. [1,30] and ref. [31] for $a \sqrt{\sigma}$ and $a / r_{0}$, respectively. We finally estimate $\Lambda_{\overline{\mathrm{MS}}} / A_{\text {phys }}$ using eq. (1.3). We show that our estimates for $\Lambda_{\overline{\mathrm{MS}}} / A_{\text {phys }}$ are compatible with the values previously obtained with other methods. This demonstrates the validity of our non-perturbative analysis.

This paper is organized as follows. In the next section, we introduce the TGF method and explain how to calculate the TGF coupling briefly. Our strategy eq. (1.3) and the details of lattice simulations are explained in section 3. $L_{\max } \Lambda_{\mathrm{TGF}}$ and $L_{\max } A_{\text {phys }}$ are presented in sections 4 and 5 , respectively. $\Lambda_{\mathrm{SF}} / \Lambda_{\mathrm{TGF}}$ and $\Lambda_{\overline{\mathrm{MS}}} / A_{\mathrm{phys}}$ are extracted in section 6. Finally we summarize this paper in the last section 7 . Our preliminary result has been presented at the Lattice conference [32].

\section{Twisted gradient flow coupling}

We use the Wilson gauge action on a $(L / a)^{4}$ lattice with twisted boundary condition:

$$
S_{\mathrm{W}}[U]=\frac{\beta}{2 N_{\mathrm{C}}} \sum_{\substack{n, \mu, \nu \\(\mu \neq \nu)}} Z_{\mu \nu}(n) \operatorname{Tr}\left[U_{\mu}(n) U_{\nu}(n+\hat{\mu}) U_{\mu}^{\dagger}(n+\hat{\nu}) U_{\nu}^{\dagger}(n)\right] .
$$

Here $U_{\mu}(n)$ is the $\mathrm{SU}\left(N_{\mathrm{C}}\right)$ link variable with periodic boundary condition. We represent the twisted boundary condition by using the twist phase $Z_{\mu \nu}(n)$. In this work, we follow 
ref. [22] and put the twisted boundary condition in the $x-y$ plane. The twist phase is defined as

$$
Z_{\mu \nu}(n)=Z_{\nu \mu}^{*}(n)= \begin{cases}\exp \left[-\frac{2 \pi i}{N_{\mathrm{C}}}\right] & \mu=1, \nu=2, \text { and } n_{1}=n_{2}=0, \\ 1 & \text { otherwise }\end{cases}
$$

in the case. The derivation of the action with the periodic variables (2.1) is given in appendix A.

We first introduce link variables $V_{\mu}(n, t)$ evolved with the gradient flow equation;

$$
\frac{\mathrm{d} V_{\mu}(n, t)}{\mathrm{d} t}=-\frac{2 N_{\mathrm{C}}}{\beta}\left\{\partial_{n, \mu} S_{\mathrm{W}}[V]\right\} V_{\mu}(n, t),\left.\quad V_{\mu}(n, t)\right|_{t=0}=U_{\mu}(n),
$$

where $t$, a fictitious time or so-called flow time, is introduced. $\partial_{n, \mu}$ is the $\mathfrak{s u}\left(N_{\mathrm{C}}\right)$-valued differential operator with respect to $V_{\mu}(n, t)$.

The twisted gradient flow (TGF) coupling $g_{\mathrm{TGF}}^{2}(1 / L)$ is defined as

$$
g_{\mathrm{TGF}}^{2}(1 / L)=\left.\mathcal{N}_{\mathrm{T}}^{-1}(c, a / L) t^{2}\langle E(t)\rangle\right|_{t=c^{2} L^{2} / 8},
$$

where $E(t)$ is a energy density made of $V_{\mu}(n, t)$. The explicit form of $E(t)$ will be given later. The vacuum expectation value $\langle E(t)\rangle$ is a renormalized quantity at the scale $1 / \sqrt{8 t}$ at any $t>0$ [20]. In a finite volume system we can use the volume size $L$ as the scale of the renormalization so we have set

$$
\frac{1}{\sqrt{8 t}}=\frac{1}{c L}
$$

in eq. (2.4). The factor $c$ is, in principle, a free parameter: a different choice of $c$ gives a different renormalization scheme. Throughout this work we choose $c=0.3$ for a reason we will state later. The normalization factor $\mathcal{N}_{\mathrm{T}}^{-1}(c, a / L)$ depends on the definition of the energy density on the lattice.

In this work, we employ the following definition for the energy density $E(t)$;

$$
E(t)=-\frac{1}{64 N_{\mathrm{C}}(L / a)^{4}} \sum_{n, \mu \neq \nu} \operatorname{Tr}\left[G_{\mu \nu}^{2}(n, t)\right]
$$

with

$$
\begin{aligned}
G_{\mu \nu}(n, t)= & Z_{\mu \nu}(n) V_{\mu}(n, t) V_{\nu}(n+\hat{\mu}, t) V_{\mu}^{\dagger}(n+\hat{\nu}, t) V_{\nu}^{\dagger}(n, t) \\
& +Z_{\mu \nu}(n-\hat{\mu}) V_{\nu}(n, t) V_{\mu}^{\dagger}(n-\hat{\mu}+\hat{\nu}, t) V_{\nu}^{\dagger}(n-\hat{\mu}, t) V_{\mu}(n-\hat{\mu}, t) \\
& +Z_{\mu \nu}(n-\hat{\mu}-\hat{\nu}) V_{\mu}^{\dagger}(n-\hat{\mu}, t) V_{\nu}^{\dagger}(n-\hat{\mu}-\hat{\nu}, t) V_{\mu}(n-\hat{\mu}-\hat{\nu}, t) V_{\nu}(n-\hat{\nu}, t) \\
& +Z_{\mu \nu}(n-\hat{\nu}) V_{\nu}^{\dagger}(n-\hat{\nu}, t) V_{\mu}(n-\hat{\nu}, t) V_{\nu}(n+\hat{\mu}-\hat{\nu}, t) V_{\mu}^{\dagger}(n, t)-\{\text { h.c. }\} . \quad(2.7)
\end{aligned}
$$

With this definition, the normalization factor $\mathcal{N}_{\mathrm{T}}(c, a / L)$, which is defined so as to match $g_{\mathrm{TGF}}^{2}(1 / L)$ with the bare coupling $g_{0}^{2}$ at the tree level of the perturbation theory, is

$$
\mathcal{N}_{\mathrm{T}}(c, a / L)=\frac{c^{4}}{128} \sum_{P}^{\prime} \exp \left[-\frac{c^{2} L^{2}}{4} \hat{P}^{2}\right] \frac{\left(\sum_{\mu} \tilde{P}_{\mu}^{2}\right)\left(\sum_{\nu} C_{\nu}^{2}\right)-\sum_{\mu}\left(\tilde{P}_{\mu} C_{\mu}\right)^{2}}{\hat{P}^{2}}
$$


where

$$
\hat{P}_{\mu}=\frac{2}{a} \sin \left[a \frac{P_{\mu}}{2}\right], \quad \tilde{P}_{\mu}=\frac{1}{a} \sin \left[a P_{\mu}\right], \quad C_{\mu}=\cos \left[a \frac{P_{\mu}}{2}\right] .
$$

The summation over $P_{\mu}$ runs

$$
P_{1,2}=\frac{2 \pi m_{1,2}}{N_{\mathrm{C}} L}, \quad 0 \leq m_{1,2} \leq \frac{N_{\mathrm{C}} L}{a}-1,
$$

for $\mu=1,2$ and

$$
P_{3,4}=\frac{2 \pi m_{3,4}}{L}, \quad 0 \leq m_{3,4} \leq \frac{L}{a}-1,
$$

for $\mu=3,4$. The prime (I) symbol on the summation indicates the exclusion of the momentum contributions with $\left(P_{1}, P_{2}\right)=(0,0)$ from the sum.

We employ $c=0.3$ throughout this work. In general, a smaller value of $c$ gives smaller statistical error. It causes, however, a larger lattice artifact. According to the previous works $[22,25], c=0.3$ gives a good compromise between these two effects. This is the reason for our choice $c=0.3$.

\section{Overview of strategy and simulation details}

Here we explain the strategy for evaluating eq. (1.3). We take the following steps.

1. We evaluate the discrete beta function $B_{s}(u)$ as a function of $u=g_{\mathrm{TGF}}^{2}(1 / L)$. It is defined as

$$
B_{s}\left(g_{\mathrm{TGF}}^{2}(1 / L)\right)=\frac{g_{\mathrm{TGF}}^{2}(s / L)-g_{\mathrm{TGF}}^{2}(1 / L)}{\log \left[s^{2}\right]},
$$

where $s$ is the scaling parameter. We extract this discrete beta function by taking the continuum limit of lattice discrete beta functions evaluated on several lattices. The details of the fitting and the analysis for the continuum limit will be explained in the next section.

2. We estimate $L_{\max } \Lambda_{\mathrm{TGF}}$ using the discrete beta function evaluated in the previous step. By fixing the scale $1 / L_{\max }$ implicitly through the value of the coupling $u^{*}=$ $g_{\text {TGF }}^{2}\left(1 / L_{\max }\right), L_{\max } \Lambda_{\mathrm{TGF}}$ can be evaluated with

$$
\begin{aligned}
c L_{\mathrm{max}} \Lambda_{\mathrm{TGF}}= & \left(b_{0} u^{*}\right)^{-\frac{b_{1}}{2 b_{0}^{2}}} \exp \left[-\frac{1}{2 b_{0} u^{*}}\right] \\
& \quad \times \exp \left[-\int_{0}^{\sqrt{u^{*}}} \mathrm{~d} \xi\left(\frac{1}{\beta_{\mathrm{TGF}}(\xi)}+\frac{1}{b_{0} \xi^{3}}-\frac{b_{1}}{\left(b_{0}\right)^{2} \xi}\right)\right] \\
\simeq & s^{n}\left(b_{0} u_{n}\right)^{-\frac{b_{1}}{2 b_{0}^{2}}} \exp \left[-\frac{1}{2 b_{0} u_{n}}\right] .
\end{aligned}
$$

Here we explicitly put $c$ on the left-hand side, which is to use the same notation as eq. (2.5) for the scale setting. The TGF coupling at scale $s^{n} / L_{\max }$ is evaluated with the following recurrence equation (step scaling),

$$
u_{i}=u_{i-1}+B_{s}\left(u_{i-1}\right) \log \left[s^{2}\right], \quad u_{0}=u^{*} .
$$


For a sufficiently small value of $u_{n}=g_{\mathrm{TGF}}^{2}\left(s^{n} / L_{\max }\right)$ we can safely use the two-loop approximation in eq. (3.2) to extract $L_{\mathrm{max}} \Lambda_{\mathrm{TGF}}$.

3. We relate the intermediate scale $1 / L_{\max }$ to a hadronic scale $A_{\text {phys }}$ in the continuum limit. We employ two hadronic scales for the consistency check; the string tension $\sqrt{\sigma}$ and the Sommer scale $r_{0}$. The lattice data of $\sqrt{\sigma}$ and $r_{0}$ are taken from refs. $[1,30]$ and [31], respectively. To outline the procedure, let us assume that $A_{\text {phys }}$ has a mass dimension one for simplicity. We interpolate each of $g_{\mathrm{TGF}}^{2}(1 / L, \beta)$ and $a A_{\mathrm{phys}}(\beta)$ as a function of bare coupling $\beta$. By keeping the coupling constant $g_{\mathrm{TGF}}^{2}\left(1 / L_{\max }, \beta^{*}\right)$ fixed to $u^{*}$ over several lattices $L_{\max } / a^{*}$, we obtain the corresponding values of $\beta^{*}$ (here to show the connection between $u^{*}$ and the lattice spacing (or bare coupling), we use $a^{*}\left(\right.$ or $\left.\beta^{*}\right)$ ). For each value of $\beta^{*}$ (thus $a^{*} / L_{\max }$ ) we have a pair of $L_{\max } / a^{*}$ and $a^{*} A_{\text {phys }}\left(\beta^{*}\right)$. We then take the continuum limit of $\left(L_{\max } / a^{*}\right)\left(a^{*} A_{\text {phys }}\right)$ as a function of $a^{*} / L_{\max }$.

4. To convert $\Lambda_{\mathrm{TGF}}$ to the $\Lambda$-parameter in the $\overline{\mathrm{MS}}$ scheme, we need the ratio $\Lambda_{\overline{\mathrm{MS}}} / \Lambda_{\mathrm{TGF}}$. We split the ratio into two pieces: $\left(\Lambda_{\overline{\mathrm{MS}}} / \Lambda_{\mathrm{SF}}\right)\left(\Lambda_{\mathrm{SF}} / \Lambda_{\mathrm{TGF}}\right)$. The value of the former factor is already known to be $\Lambda_{\overline{\mathrm{MS}}} / \Lambda_{\mathrm{SF}}=0.48811$ (1) [12], but the latter is not known in the literature. We therefore calculate $\Lambda_{\mathrm{SF}} / \Lambda_{\mathrm{TGF}}$ numerically via the one-loop relation between $g_{\mathrm{SF}}^{2}$ and $g_{\mathrm{TGF}}^{2}$ at the same renormalization scale $1 / L$. To obtain the one-loop relation, we calculate the couplings with lattice simulations in the weak coupling region.

5. Finally we combine all pieces obtained above to have

$$
\frac{\Lambda_{\overline{\mathrm{MS}}}}{\sqrt{\sigma}}=\frac{\Lambda_{\overline{\mathrm{MS}}}}{\Lambda_{\mathrm{SF}}} \frac{\Lambda_{\mathrm{SF}}}{\Lambda_{\mathrm{TGF}}} \frac{L_{\mathrm{max}} \Lambda_{\mathrm{TGF}}}{L_{\max } \sqrt{\sigma}}, \quad r_{0} \Lambda_{\overline{\mathrm{MS}}}=\frac{\Lambda_{\overline{\mathrm{MS}}}}{\Lambda_{\mathrm{SF}}} \frac{\Lambda_{\mathrm{SF}}}{\Lambda_{\mathrm{TGF}}} \frac{L_{\mathrm{max}} \Lambda_{\mathrm{TGF}}}{L_{\max } / r_{0}} .
$$

The TGF couplings on the lattice in the steps 1-3 explained above are evaluated on five lattices with $L / a=12,16,18,24$ and 36 . We use the heat-bath method introduced by Fabricius and Haan [33] to increase the acceptance ratio. We accumulate configurations as listed in table 10 in appendix B. Each configuration is separated by 100 sweeps. The TGF couplings, we computed, are listed in table 1, of which error is statistical one and estimated by taking the autocorrelation into account with the procedure proposed in ref. [34]. ${ }^{1}$ We take several values for the bare coupling $\beta=6 / g_{0}^{2}$ on each lattice to take the continuum limit.

On the other hand, simulations in the weak coupling region have been done on four lattices $L / a=8,10,12$ and 16 , with three values of the bare coupling $\beta=40,60$ and 80 . We use the same plaquette gauge action with the $O(a)$-improvement boundary correction and the SF boundary condition $[4,35]$ to calculate $g_{\mathrm{SF}}^{2}$. The error of the coupling from these data is estimated with the Jackknife method after binning data into 10 bins. We execute $O\left(10^{6}\right)-O\left(10^{7}\right)$ sweeps for each parameter. The SF coupling is evaluated every sweep and the TGF coupling is evaluated every 100 sweeps.

\footnotetext{
${ }^{1}$ We observed long autocorrelations for some of the parameter sets so we increased the statistics for them. We leave the identification of the source of this behavior for future study.
} 


\begin{tabular}{|c|c|c|c|c|c|c|}
\hline \multirow[b]{2}{*}{$\beta$} & \multicolumn{5}{|c|}{$g_{\mathrm{TGF}}^{2}(1 / L, \beta)$} & \multirow[b]{2}{*}{$L / a$} \\
\hline & 12 & 16 & 18 & 24 & 36 & \\
\hline 6.11 & $6.9717(35)$ & & & & & \\
\hline 6.13 & $6.6924(34)$ & & & & & \\
\hline 6.15 & $6.4394(34)$ & & & & & \\
\hline 6.17 & $6.1930(33)$ & & & & & \\
\hline 6.20 & $5.8715(38)$ & & & & & \\
\hline 6.25 & $5.4287(42)$ & & & & & \\
\hline 6.30 & & $7.0234(91)$ & & & & \\
\hline 6.31 & & $6.8951(95)$ & & & & \\
\hline 6.33 & $4.8848(34)$ & $6.6495(96)$ & & & & \\
\hline 6.36 & & $6.2972(90)$ & & & & \\
\hline 6.38 & & $6.102(11)$ & $7.082(14)$ & & & \\
\hline 6.40 & $4.5129(29)$ & $5.892(12)$ & $6.848(14)$ & & & \\
\hline 6.42 & $4.4250(27)$ & $5.6829(75)$ & $6.595(12)$ & & & \\
\hline 6.45 & $4.3034(29)$ & $5.4760(85)$ & $6.263(12)$ & & & \\
\hline 6.47 & & & $6.034(17)$ & & & \\
\hline 6.49 & & & $5.868(14)$ & & & \\
\hline 6.50 & $4.1052(26)$ & $5.118(13)$ & $5.734(14)$ & & & \\
\hline 6.60 & $3.7783(18)$ & $4.5767(39)$ & $5.049(14)$ & $6.967(27)$ & & \\
\hline
\end{tabular}

Table 1. TGF coupling on each $L / a$ and $\beta$.

The error propagation of the statistical error on non-primary observables, such as the discrete beta function in the continuum limit, is estimated by a random re-sampling method. For the re-sampling, we assume the primary data in table 1 satisfies Gaussian distribution with the width of the measured statistical error.

\section{TGF running coupling constant and $L_{\max } \Lambda_{\mathrm{TGF}}$}

To extract the discrete beta function eq. (3.1), we take the continuum limit of the lattice discrete beta function defined by

$$
B_{s}^{\mathrm{LAT}}\left(g_{\mathrm{TGF}}^{2}(1 / L, \beta)\right)=\frac{g_{\mathrm{TGF}}^{2}(s / L, \beta)-g_{\mathrm{TGF}}^{2}(1 / L, \beta)}{\log \left[s^{2}\right]} .
$$

We use $s=3 / 2$ as the scaling parameter. To take the continuum limit of eq. (4.1), the value of $g_{\mathrm{TGF}}^{2}(1 / L, \beta)$ is kept fixed at $g_{\mathrm{TGF}}^{2}(1 / L, \beta)=u$ as the renormalization condition irrespective of $\beta$. This implies that the physical length $L$ is fixed. The lattice discrete beta function is evaluated using eq. (4.1) by substituting the data of table 1 . In the following we fit the lattice discrete beta function to a polynomial function and investigate the systematic errors coming from the fitting. 


\begin{tabular}{|r|ccccll|}
\hline & \multicolumn{5}{|c|}{$g_{\text {TGF }}^{2}(1 / L, \beta)$} & \\
\multicolumn{1}{|c|}{16} & 18 & 24 & 36 & $L / a$ \\
\hline 6.62 & 12 & $4.4730(40)$ & & $6.705(21)$ & \\
6.65 & $3.6355(17)$ & $4.3581(56)$ & $4.799(15)$ & $6.352(25)$ & \\
6.75 & $3.3836(18)$ & $3.9874(69)$ & $4.3092(87)$ & $5.4640(88)$ & \\
6.80 & & $3.8284(31)$ & & $5.1284(90)$ & \\
6.86 & $3.1542(15)$ & $3.6604(33)$ & & $4.8079(69)$ & \\
6.90 & & & & $4.6334(48)$ & $7.009(19)$ \\
6.94 & & & $4.4546(67)$ & $6.590(26)$ \\
6.97 & & & $4.3189(55)$ & $6.317(28)$ \\
7.00 & $2.9041(14)$ & $3.3168(75)$ & $3.5318(73)$ & $4.2230(44)$ & $5.983(14)$ \\
7.05 & & $3.2164(24)$ & & $4.0409(43)$ & \\
7.13 & & & & $3.7924(38)$ & $5.0811(92)$ \\
7.18 & & & & $3.6537(34)$ & $4.8281(68)$ \\
7.25 & $2.5489(12)$ & $2.8649(40)$ & $3.0163(57)$ & $3.4803(31)$ & $4.4890(60)$ \\
7.40 & & & & $3.1596(28)$ & $3.9612(56)$ \\
7.50 & $2.2783(11)$ & $2.5122(41)$ & $2.6370(57)$ & $2.9831(27)$ & $3.6693(50)$ \\
7.75 & $2.06258(94)$ & $2.2627(32)$ & $2.3452(47)$ & $2.6125(23)$ & $3.1269(34)$ \\
8.00 & $1.88717(88)$ & $2.0469(36)$ & $2.1238(42)$ & $2.3323(21)$ & $2.7269(27)$ \\
8.25 & $1.74054(80)$ & $1.8722(30)$ & $1.9380(35)$ & $2.1078(17)$ & $2.4235(27)$ \\
8.50 & $1.61638(71)$ & $1.7312(26)$ & $1.7868(34)$ & $1.9273(11)$ & $2.1836(18)$ \\
9.00 & $1.41544(65)$ & $1.4971(24)$ & $1.5438(39)$ & $1.64590(88)$ & $1.8261(15)$ \\
9.50 & $1.26024(62)$ & $1.3242(22)$ & $1.3576(30)$ & $1.43870(82)$ & $1.5689(12)$ \\
10.00 & $1.13594(49)$ & $1.1879(16)$ & $1.2134(17)$ & $1.27733(67)$ & $1.3803(12)$ \\
\hline
\end{tabular}

Table 1. (cont'd) TGF coupling on each $L / a$ and $\beta$.

We use the following polynomial function of $a / L$ and $u$ for the fitting;

$$
B_{3 / 2}^{\mathrm{LAT}}(u, a / L)=\sum_{j=0}^{N_{\max }}\left[c_{j}+d_{j}\left(\frac{a}{L}\right)^{2}\right] u^{j+2} .
$$

Here $c_{0}=-b_{0}$ and $c_{1}=b_{0}^{2} \log \left[s^{2}\right]-b_{1}$ are fixed to the analytical values from the twoloop perturbation. $N_{\max }$ is an order of truncation to investigate the uncertainty from the fit-ansatz as a polynomial in $u$. We compare the parameters fitted with $N_{\max }=3$ and $N_{\max }=4$ to see the truncation error in $u$. The lattice cut-off dependence is incorporated with $d_{j}$ for $O\left(a^{2} / L^{2}\right)$ effects. We also investigate the fit range dependence in $u$ by varying the upper limit around $u^{\max } \sim 6.263-6.848$ together with the truncation effect in $c_{j}$ and $d_{j}$. To use interpolated data for the analysis, we set $u^{\max }$ defined by

$$
u^{\max }=\min _{L / a}\left(\max _{\beta} g_{\mathrm{TGF}}^{2}(1 / L, \beta)\right),
$$

where $\max _{\beta}$ takes the maximum value of $g_{\mathrm{TGF}}^{2}$ among different values of $\beta$ with fixed $L / a$. 


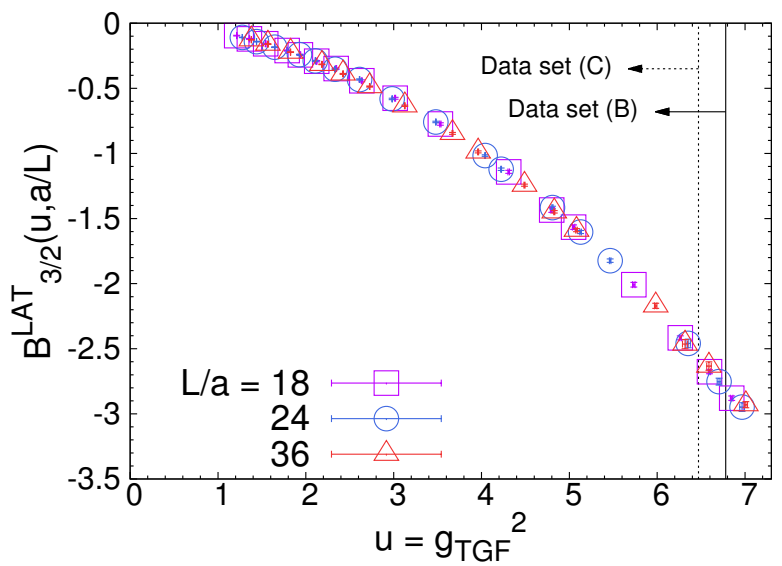

Figure 1. The discrete beta function on each lattice size.

\begin{tabular}{|c|cc|cc|}
\hline Data set & \multicolumn{2}{|c|}{ Set (A) } & \multicolumn{2}{c|}{ Set (A) with $d_{N_{\max }}=0$} \\
$N_{\max }$ & 3 & 4 & 3 & 4 \\
\hline$c_{2}$ & $0.000657(26)$ & $0.00143(15)$ & $0.000644(26)$ & $0.00145(15)$ \\
$c_{3}$ & $-0.0000613(39)$ & $-0.000337(53)$ & $-0.0000589(39)$ & $-0.000344(52)$ \\
$c_{4}$ & - & $0.0000237(46)$ & - & $0.0000243(45)$ \\
$d_{0}$ & $-0.79(58)$ & $2.1(1.3)$ & $1.88(27)$ & $1.49(71)$ \\
$d_{1}$ & $1.92(44)$ & $-1.6(1.5)$ & $-0.25(15)$ & $-0.84(67)$ \\
$d_{2}$ & $-0.54(10)$ & $0.53(57)$ & $-0.019(16)$ & $0.22(17)$ \\
$d_{3}$ & $0.0389(75)$ & $-0.072(91)$ & - & $-0.021(13)$ \\
$d_{4}$ & - & $0.0030(52)$ & - & - \\
\hline$\chi^{2} / \mathrm{DoF}$ & $2.39(59)$ & $1.60(52)$ & $3.11(64)$ & $1.56(51)$ \\
\hline
\end{tabular}

Table 2. Fit results with the set (A) for the discrete beta function.

We have three data sets [(A), (B) and (C)] for the fitting stability analysis.

- Set (A): all data pairs at $L / a=(12,18),(16,24)$, or $(24,36)$ in each $\beta$ row listed in table 1 . This corresponds to $u^{\max } \simeq 6.848$.

- Set (B): following data pairs are excluded from the set (A): $\beta=6.40$ for $L / a=$ $(12,18), \beta=6.60$ for $L / a=(16,24)$, and $\beta=6.90$ for $L / a=(24,36)$. This corresponds to $u^{\max } \simeq 6.590$.

- Set (C): following data pairs are excluded from the set (B): $\beta=6.42$ for $L / a=$ $(12,18), \beta=6.62$ for $L / a=(16,24)$, and $\beta=6.94$ for $L / a=(24,36)$. This corresponds to $u^{\max } \simeq 6.263$.

Figure 1 shows the lattice discrete beta function. The set (A) uses all data points in the figure. For the set (B) and the set (C), we use all data points below the solid vertical line and dotted vertical line, respectively. We fit data $\left(B_{3 / 2}^{\mathrm{LAT}}(u, a / L), u, a / L\right)$ simultaneously in 


\begin{tabular}{|c|cc|cc|}
\hline Data set & \multicolumn{2}{|c|}{ Set (B) } & \multicolumn{2}{c|}{ Set (B) with $d_{N_{\max }}=0$} \\
$N_{\max }$ & 3 & 4 & 3 & 4 \\
\hline$c_{2}$ & $0.000755(34)$ & $0.00110(21)$ & $0.000740(34)$ & $0.00110(20)$ \\
$c_{3}$ & $-0.0000802(57)$ & $-0.000208(76)$ & $-0.0000774(56)$ & $-0.000211(73)$ \\
$c_{4}$ & - & $0.0000117(69)$ & - & $0.0000119(66)$ \\
$d_{0}$ & $-0.09(64)$ & $1.0(1.4)$ & $1.63(29)$ & $0.84(82)$ \\
$d_{1}$ & $1.17(52)$ & $-0.2(1.7)$ & $-0.29(17)$ & $0.00(83)$ \\
$d_{2}$ & $-0.37(13)$ & $0.04(66)$ & $-0.001(20)$ & $-0.03(23)$ \\
$d_{3}$ & $0.0287(97)$ & $-0.01(11)$ & - & $0.000(19)$ \\
$d_{4}$ & - & $0.0008(67)$ & - & - \\
\hline$\chi^{2} / \mathrm{DoF}$ & $1.42(52)$ & $1.41(54)$ & $1.66(54)$ & $1.36(52)$ \\
\hline
\end{tabular}

Table 3. Fit results with the set (B) for the discrete beta function.

\begin{tabular}{|c|cc|cc|}
\hline Data set & \multicolumn{2}{|c|}{ Set (C) } & \multicolumn{2}{c|}{ Set $(\mathrm{C})$ with $d_{N_{\max }}=0$} \\
$N_{\max }$ & 3 & 4 & 3 & 4 \\
\hline$c_{2}$ & $0.000809(44)$ & $0.00100(23)$ & $0.000793(43)$ & $0.00099(22)$ \\
$c_{3}$ & $-0.0000912(80)$ & $-0.000165(90)$ & $-0.0000884(78)$ & $-0.000160(84)$ \\
$c_{4}$ & - & $0.0000070(86)$ & - & $0.0000066(80)$ \\
$d_{0}$ & $0.43(75)$ & $0.6(1.8)$ & $1.56(34)$ & $0.87(93)$ \\
$d_{1}$ & $0.69(63)$ & $0.4(2.1)$ & $-0.30(21)$ & $0.11(95)$ \\
$d_{2}$ & $-0.26(16)$ & $-0.22(91)$ & $0.006(27)$ & $-0.08(27)$ \\
$d_{3}$ & $0.022(13)$ & $0.03(16)$ & - & $0.007(23)$ \\
$d_{4}$ & - & $-0.002(11)$ & - & - \\
\hline$\chi^{2} / \mathrm{DoF}$ & $1.36(52)$ & $1.44(55)$ & $1.41(52)$ & $1.39(53)$ \\
\hline
\end{tabular}

Table 4. Fit results with the set (C) for the discrete beta function.

$u$ and $a / L$ by taking the correlation among $u$ 's and $B_{3 / 2}^{\mathrm{LAT}}(u, a / L)$ 's into account [36] for each data set. The continuum limit is obtained by dropping the $d_{j}$ terms.

Tables 2, 3, and 4 show the fit results in each case. As seen from the results in the set (A), the discrepancy for $c_{j}$ between $N_{\max }=3$ and 4 is rather large indicating the uncontrolled systematic error in the polynomial fit at these polynomial order. We cannot determine the higher order terms using data with large statistical error in the strong coupling region. We observe that the discrepancy in $c_{j}$ between $N_{\max }=3$ and 4 for sets (B) and (C) is smaller than that for the set (A). The coefficients $c_{j}$ in the set (C) are consistent between $N_{\max }=3$ and 4 , though there are large statistical errors. The truncation error in $u$ seems to be controlled in the fitting range of $(\mathrm{C})$.

We also investigate the stability on fitting for $d_{j} \quad\left(j<N_{\max }\right)$ by dropping the parameter $d_{N_{\max }}$. For the set (A), setting $d_{N_{\max }}=0$ in the fit-ansatz largely affects on the determination on the coefficients $d_{j} \quad\left(j<N_{\max }\right)$. For the sets (B) and (C), however, the stability on $d_{j}$ improves. Although the coefficients $d_{j}$ have large statistical errors, the consistency in 


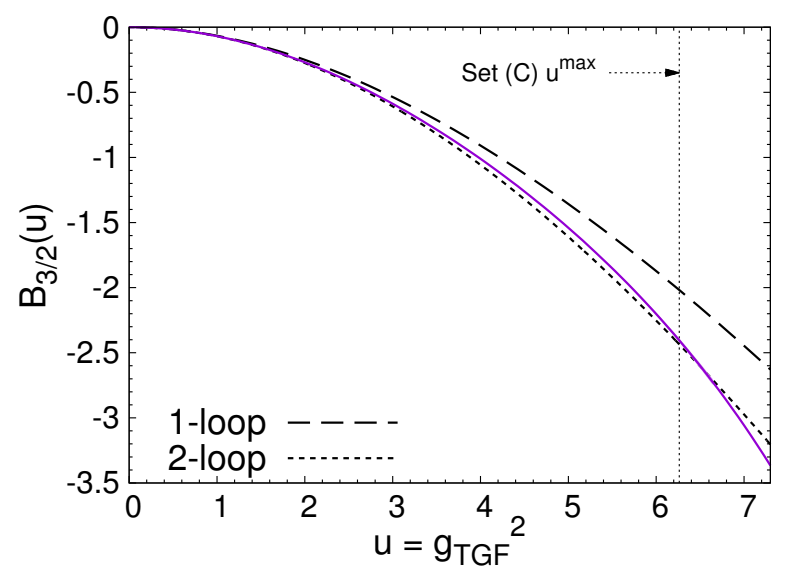

Figure 2. The discrete beta function in the continuum limit (solid purple line) obtained with $N_{\max }=3$ from the set $(\mathrm{C})$ together with the one- and two-loop analytic results. The statistical error band (light purple shade) for the result is underlaid behind the solid purple line, but is almost identical to the width of the line.

the set $(\mathrm{C})$ is better than that in the set $(\mathrm{B})$. Moreover we observe that the truncation of $d_{N_{\max }}$ does not disturb the coefficients $c_{2}$ and $c_{3}$, which indicates that the continuum limit is insensitive to the lattice cut-off effect with the fit-ansatz we employed. In the rest of the analysis, we always use results obtained with $d_{N_{\max }} \neq 0$ without explicitly mentioning.

Because of these observations we adopt the results obtained with $N_{\max }=3$ from the data set $(\mathrm{C})$ as the central value. The other results obtained from the data set (B) and (C) are used to investigate the systematic error on the $\Lambda$-parameter. Figure 2 shows the discrete beta function in the continuum limit obtained with $N_{\max }=3$ from the set (C).

We evaluate $L_{\text {max }} \Lambda_{\text {TGF }}$ according to the step 2 in section 3. Eleven values for $u^{*}$ are taken from 6.00 to 6.25 in steps of 0.025 to fix the intermediate scale $L_{\max }$. The fluctuations coming from the different choice of $L_{\max }$, fit-ansatz and data set will be used to estimate the systematic errors of the final results of $r_{0} \Lambda_{\overline{\mathrm{MS}}}$ and $\Lambda_{\overline{\mathrm{MS}}} / \sqrt{\sigma}$ in section 5 . The number of steps $n$ to evolve eq. (3.3) is $n=400$, where $u_{n=400}$ is sufficiently small to utilize eq. (3.2). The values of $c L_{\max } \Lambda_{\mathrm{TGF}}$ for each $u^{*}$ are tabulated in table 5 .

\section{Physical scale in terms of $L_{\max }$}

As described in section 3, the hadronic scales, the string tension $\sqrt{\sigma}$ and the Sommer scale $r_{0}$, have to be determined in terms of $L_{\max } \cdot a \sqrt{\sigma}$ and $r_{0} / a$ with the plaquette gauge action in large physical volumes have been determined at $\beta \in[5.65,6.515]$ in refs. $[1,30]$ and $\beta \in[5.70,6.692]$ in ref. [31], respectively. To relate the intermediate scale $L_{\max } / a$ and the physical scales $a A_{\text {phys }}\left(=a \sqrt{\sigma}\right.$ or $\left.a / r_{0}\right)$ at the same lattice cut-off " $a$ ", we need the bare coupling constant $g_{0}^{2}$ dependence (or $\beta$ dependence) of $g_{\mathrm{TGF}}^{2}\left(1 / L_{\max }, \beta\right)$ and $a A_{\text {phys }}(\beta)$.

Using the values of $a A_{\text {phys }}\left(\beta^{*}\right)$ at a fixed value $g_{\text {TGF }}^{2}\left(a^{*} / L_{\max }, \beta^{*}\right)=u^{*}$ on several lattice sizes are obtained, we can take the continuum limit for $L_{\max } A_{\text {phys }}$ as follows:

$$
L_{\mathrm{max}} A_{\mathrm{phys}}=\left.\lim _{a^{*} / L_{\max } \rightarrow 0}\left[\left(\frac{L_{\mathrm{max}}}{a^{*}}\right)\left(a^{*} A_{\mathrm{phys}}\right)\right]\right|_{\text {fixed } g_{\mathrm{TGF}}^{2}=u^{*}} .
$$




\begin{tabular}{|c|c|c|c|c|}
\hline \multirow[b]{3}{*}{$u^{*}=g_{\mathrm{TGF}}^{2}\left(1 / L_{\max }\right)$} & \multicolumn{4}{|c|}{$c L_{\max } \Lambda_{\mathrm{TGF}}$} \\
\hline & \multicolumn{2}{|c|}{ Set $(B)$} & \multicolumn{2}{|c|}{ Set $(\mathrm{C})$} \\
\hline & $N_{\max }=3$ & $N_{\max }=4$ & $N_{\max }=3$ & $N_{\max }=4$ \\
\hline 6.000 & $0.5791(67)$ & $0.6142(229)$ & $0.5845(74)$ & $0.6040(253)$ \\
\hline 6.025 & $0.5812(67)$ & $0.6164(230)$ & $0.5865(74)$ & $0.6061(254)$ \\
\hline 6.050 & $0.5832(67)$ & $0.6186(231)$ & $0.5885(74)$ & $0.6082(256)$ \\
\hline 6.075 & $0.5852(67)$ & $0.6208(232)$ & $0.5904(74)$ & $0.6103(257)$ \\
\hline 6.100 & $0.5872(67)$ & $0.6230(233)$ & $0.5923(74)$ & $0.6123(259)$ \\
\hline 6.125 & $0.5892(68)$ & $0.6251(234)$ & $0.5942(74)$ & $0.6143(260)$ \\
\hline 6.150 & $0.5912(68)$ & $0.6273(235)$ & $0.5961(74)$ & $0.6163(262)$ \\
\hline 6.175 & $0.5931(68)$ & $0.6294(236)$ & $0.5980(73)$ & $0.6183(264)$ \\
\hline 6.200 & $0.5950(68)$ & $0.6315(237)$ & $0.5998(73)$ & $0.6203(265)$ \\
\hline 6.225 & $0.5969(68)$ & $0.6336(238)$ & $0.6020(73)$ & $0.6222(267)$ \\
\hline 6.250 & $0.5988(68)$ & $0.6357(239)$ & $0.6037(73)$ & $0.6242(269)$ \\
\hline
\end{tabular}

Table 5. $c L_{\max } \Lambda_{\mathrm{TGF}}$ for each $u^{*}$.

To take the continuum limit of the hadronic scale $a A_{\text {phys }}$ reliably, $g_{\mathrm{TGF}}^{2}\left(1 / L_{\mathrm{max}}, \beta\right)$ should be precisely evaluated in the scaling region of $a A_{\text {phys }}$ on several lattice sizes $L_{\max } / a$ with sufficiently large $u^{*}$. This condition is satisfied with our data at $L_{\max } / a=12,16$ and 18 , where the large enough TGF couplings $g_{\mathrm{TGF}}^{2}\left(1 / L_{\max }, \beta\right)=u^{*}$ and $a A_{\text {phys }}$ in the scaling region are available in the ranges $\beta \in[6.11,6.515]$ for $a \sqrt{\sigma}$ and $\beta \in[6.11,6.92]$ for $a / r_{0}$, respectively. Therefore we can take any renormalization condition $u^{*}$ in this region and we employ several different values from $u^{*}=6.00-6.25$, of which upper limit is below $u^{\max } \simeq$ 6.263 of the set $(\mathrm{C})$, to see that the final results do not depend on the choice of $u^{*}$ indeed.

Let us start with interpolation of $g_{\mathrm{TGF}}^{2}\left(1 / L_{\max }, \beta\right), a \sqrt{\sigma}$ and $a / r_{0}$ as functions of $g_{0}^{2}$ separately in the following. Then we combine the interpolated results to take the continuum limit using eq. (5.1).

To interpolate $g_{\mathrm{TGF}}^{2}\left(1 / L_{\max }, \beta\right)$, we use all data satisfying the following conditions; $\beta \in[6.11,6.60], g_{\text {TGF }}^{2}>5.0$, and $L_{\text {max }} / a=12,16$ or 18 in table 1 . In this $\beta$ region, both the Sommer scale and the string tension are available in large volumes. We interpolate the data using the following function;

$$
g_{\mathrm{TGF}}^{2}\left(1 / L_{\max }, \beta\right)=g_{0}^{2} \frac{1}{1+\sum_{k=1}^{3} c_{k} g_{0}^{2 k}} .
$$

Figure 3 shows the fit result, and table 6 shows the parameters obtained. Solving

$$
g_{\mathrm{TGF}}^{2}\left(1 / L_{\max }, \beta^{*}\right)=u^{*}
$$

at each $u^{*}$ for $\beta^{*}$ using eq. (5.2), we obtain $\beta^{*}$ as shown in table 11 in appendix C. 


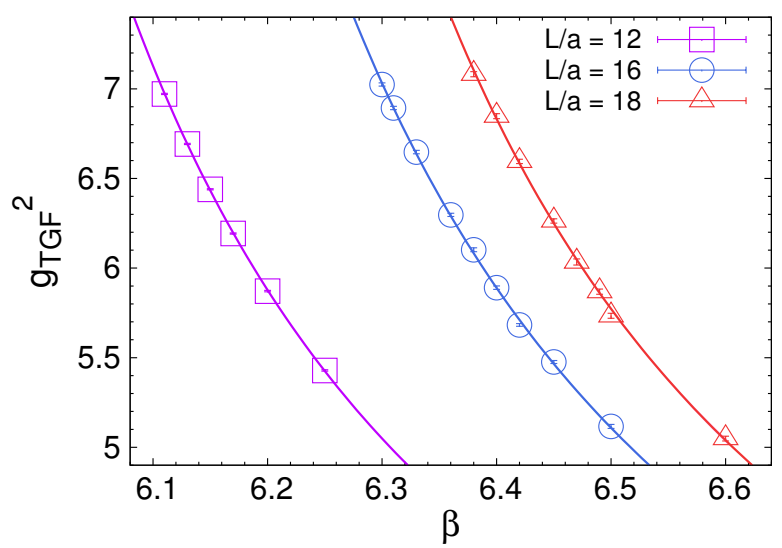

Figure 3. $g_{\mathrm{TGF}}^{2}(1 / L, \beta)$ vs $\beta$ at each lattice size. The solid lines show the fit results with eq. (5.2).

\begin{tabular}{|cclcc|}
\hline$L / a$ & $c_{1}$ & $c_{2}$ & $c_{3}$ & $\chi^{2} / \mathrm{DoF}$ \\
\hline 12 & $-3.03(0.78)$ & $5.25(1.60)$ & $-3.12(0.82)$ & $3.2(2.2)$ \\
16 & $-2.95(1.05)$ & $5.06(2.24)$ & $-3.07(1.19)$ & $3.6(1.6)$ \\
18 & $-3.23(1.22)$ & $5.69(2.62)$ & $-3.44(1.41)$ & $2.9(1.7)$ \\
\hline
\end{tabular}

Table 6. Fitted parameters for eq. (5.2) at each lattice size.

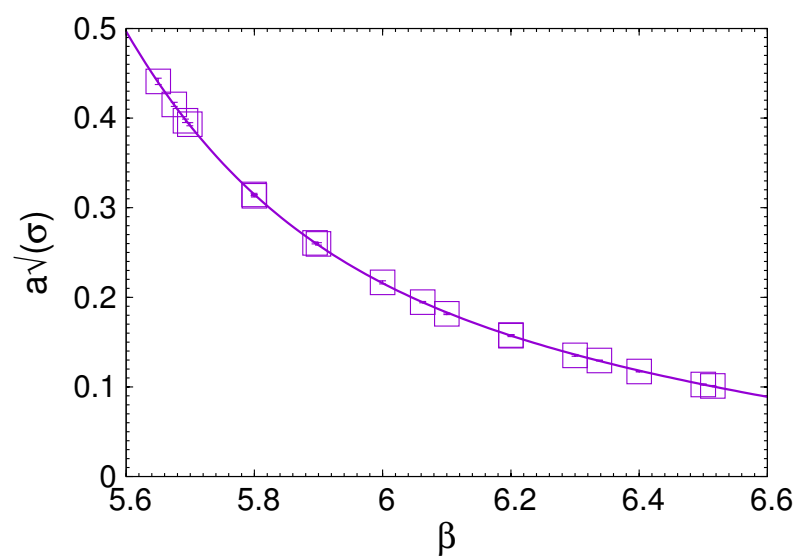

Figure 4. The $\beta$ dependence of the string tension $a \sqrt{\sigma}$.

Interpolating the data from refs. $[1,30]$ for $a \sqrt{\sigma}$ as a function of $x=g_{0}^{2}$, we obtain

$$
\begin{aligned}
(a \sqrt{\sigma})(x) & =f(x)\left(-12536(1651)+40079(5022) x-42596(5087) x^{2}+15145(1716) x^{3}\right), \\
f(x) & =\left(b_{0} x\right)^{-\frac{b_{1}}{2 b_{0}^{2}}} \exp \left[-\frac{1}{2 b_{0} x}\right],
\end{aligned}
$$

with $\chi^{2} / \mathrm{DoF}=1.15(67)$. As plotted in figure 4, eq. (5.3) smoothly interpolates the data in the scaling region $\beta \in[6.11,6.515]$. Substituting $\beta^{*}$ from table 11 into eq. (5.3), and multiplying $L_{\max } / a^{*}$ which corresponds to $\beta^{*}$ on it, we obtain $L_{\max } \sqrt{\sigma}$ at each $u^{*}$. Table 12 in appendix $\mathrm{C}$ shows the values of $L_{\max } \sqrt{\sigma}$ before taking the continuum limit. The cut-off 

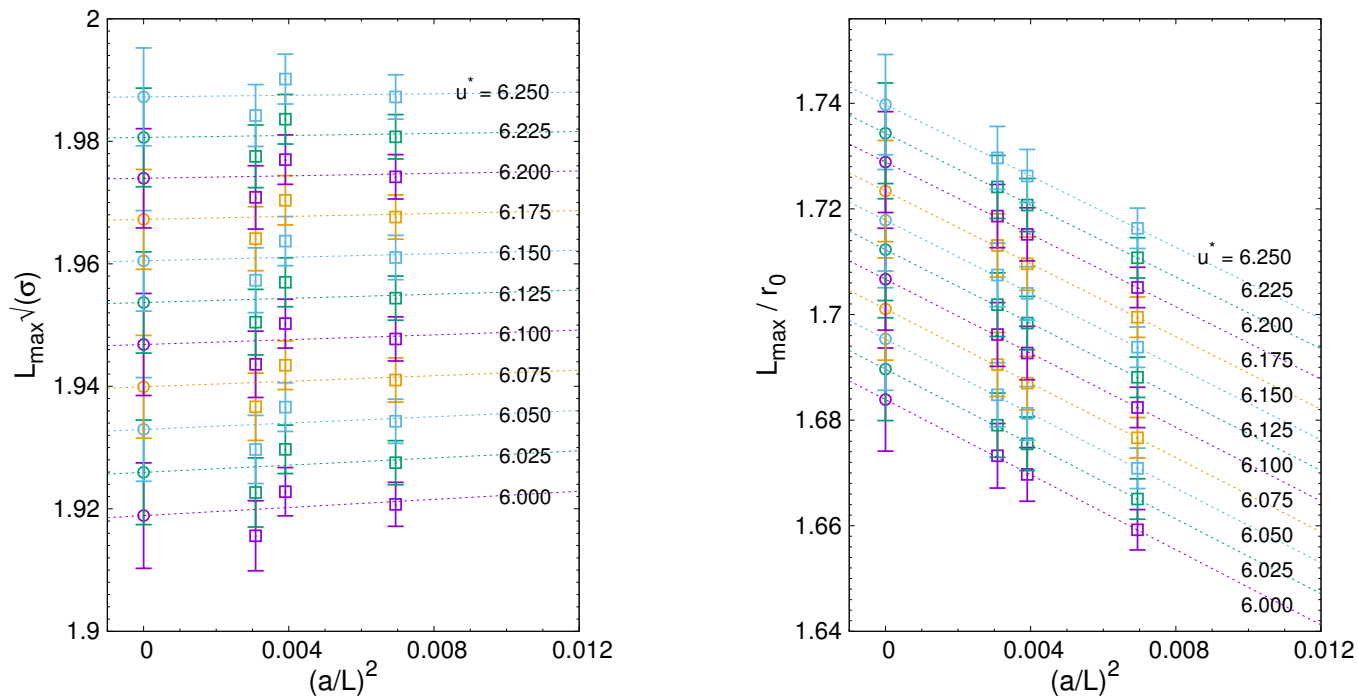

Figure 5. $(a / L)^{2}$ dependence of $L_{\max } \sqrt{\sigma}$ (left) and $L_{\max } / r_{0}$ (right) for each $u^{*}$. The lines show the liner extrapolation to the continuum limit.

\begin{tabular}{|cll|}
\hline$u^{*}=g_{\mathrm{TGF}}^{2}\left(1 / L_{\max }\right)$ & $L_{\max } \sqrt{\sigma}$ & $L_{\max } / r_{0}$ \\
\hline 6.000 & $1.9189(86)$ & $1.6839(98)$ \\
6.025 & $1.9260(85)$ & $1.6896(97)$ \\
6.050 & $1.9330(85)$ & $1.6954(97)$ \\
6.075 & $1.9399(84)$ & $1.7010(97)$ \\
6.100 & $1.9468(83)$ & $1.7067(97)$ \\
6.125 & $1.9537(83)$ & $1.7123(96)$ \\
6.150 & $1.9605(82)$ & $1.7179(96)$ \\
6.175 & $1.9673(82)$ & $1.7234(96)$ \\
6.200 & $1.9740(81)$ & $1.7289(96)$ \\
6.225 & $1.9806(80)$ & $1.7343(95)$ \\
6.250 & $1.9873(80)$ & $1.7397(95)$ \\
\hline
\end{tabular}

Table 7. $L_{\max } \sqrt{\sigma}$ and $L_{\max } / r_{0}$ for each $u^{*}$ in the continuum limit.

dependence of $L_{\max } \sqrt{\sigma}$ for each $u^{*}$ is shown in the left panel of figure 5 . We extrapolate them linearly in $a^{2} / L^{2}$ to the continuum limit (circles). $\chi^{2} /$ DoF $\sim 1$ are obtained from the extrapolation. Since the resulting value of the $\chi^{2} / \mathrm{DoF}$ is reasonable, and naive order estimation of contribution from $(a / L)^{4}$ is much smaller than the statistical error, we neglect the $O\left(a^{4} / L^{4}\right)$ uncertainty in the continuum extrapolation. The values in the continuum limit are tabulated in the middle column of table 7 .

We analyze $L_{\max } / r_{0}$ similarly to the case of $L_{\max } \sqrt{\sigma}$. The interpolating formula is

$$
\begin{aligned}
\frac{a}{r_{0}}(x)=f(x)( & 23977(12145)-106580(50576) x \\
& \left.+178094(78876) x^{2}-132318(54603) x^{3}+36906(14157) x^{4}\right),
\end{aligned}
$$




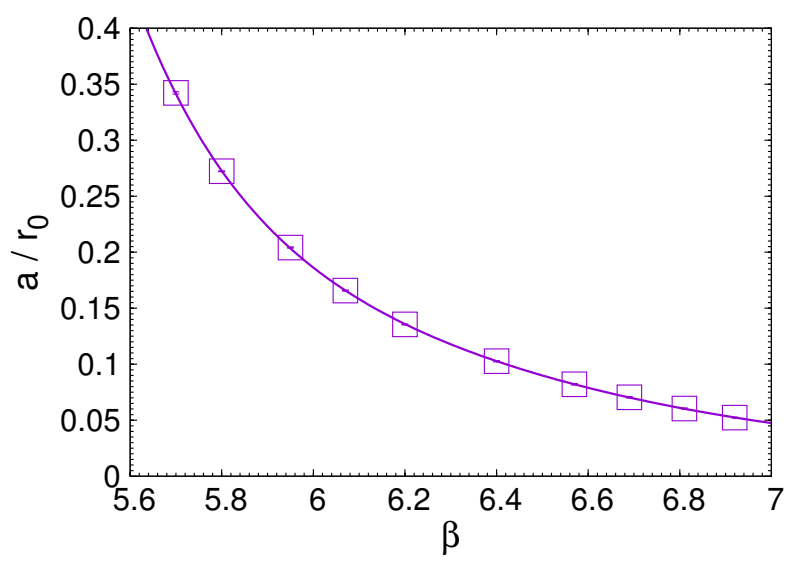

Figure 6. The $\beta$ dependence of the Sommer scale $a / r_{0}$.

with $\chi^{2} / \mathrm{DoF}=1.14(1.14)$ (figure 6 shows the interpolation in the scaling region). We list the values of $L_{\max } / r_{0}$ at each renormalization condition $u^{*}$ with finite lattice cut-off in table 13 in appendix C. The cut-off dependence and the values in the continuum limit are shown in the right panel of figure 5 and the right column in table 7 , respectively.

\section{$6 \quad \Lambda$-parameter ratio $\Lambda_{\mathrm{SF}} / \Lambda_{\mathrm{TGF}}$ and $\Lambda_{\overline{\mathrm{MS}}}$}

To move from the TGF scheme to the $\overline{\mathrm{MS}}$ scheme, we need the $\Lambda$-parameter ratio $\Lambda_{\overline{\mathrm{MS}}} / \Lambda_{\mathrm{TGF}}$. Usually the ratio is calculated with the one-loop perturbation theory but the value is not yet available at the present time, while there is an ongoing project [28] of the perturbative calculation. As we already know the ratio $\Lambda_{\overline{\mathrm{MS}}} / \Lambda_{\mathrm{SF}}$ [12], what we have to calculate is the ratio $\Lambda_{\mathrm{SF}} / \Lambda_{\mathrm{TGF}}$. Since both $g_{\mathrm{SF}}^{2}$ and $g_{\mathrm{TGF}}^{2}$ can be evaluated on the lattice with the same cut-off and with the renormalization scale (that is, $a$ and $L$ are the same), we can evaluate them with the Monte Carlo simulation on the lattice. We employ the two-loop formula [4, 35],

$$
c_{\mathrm{t}}\left(g_{0}\right)=1-0.08900(5) g_{0}^{2}-0.0294(3) g_{0}^{4},
$$

for the $O(a)$-improvement boundary correction in the $\mathrm{SF}$ simulations so that $g_{\mathrm{SF}}^{2}$ is $O(a)$ improved at the two-loop level.

Let us denote the SF and TGF couplings at the gauge coupling $\beta$ on a finite box $(L / a)^{4}$ by $g_{\mathrm{SF}}^{2}(a / L, \beta)$ and $g_{\mathrm{TGF}}^{2}(a / L, \beta)$, respectively. In a weak coupling region, these couplings are related through

$$
\frac{g_{\mathrm{SF}}^{2}(a / L, \beta)}{g_{\mathrm{TGF}}^{2}(a / L, \beta)}=1+c_{\mathrm{g}}(a / L) g_{\mathrm{TGF}}^{2}(a / L, \beta)+\cdots .
$$

We extract the value of $c_{g}(a / L)$ by investigating $g_{\text {TGF }}^{2}(a / L, \beta)$ dependence of the ratio (6.2). Both couplings $g_{\mathrm{TGF}}^{2}$ and $g_{\mathrm{SF}}^{2}$ are numerically evaluated at $\beta=40,60$ and 80 on $L / a=8$, 10, 12 and 16 lattices. Since the TGF scheme is automatically free from $O(a)$ errors and $g_{\mathrm{SF}}$ is $O(a)$-improved, the $a / L$ dependence of $c_{\mathrm{g}}(a / L)$ should be

$$
c_{\mathrm{g}}(a / L)=c_{\mathrm{g}}^{(0)}+c_{\mathrm{g}}^{(1)}\left(\frac{a}{L}\right)^{2}+\cdots .
$$




\begin{tabular}{|cccl|llll|}
\hline$L / a$ & $\beta$ & $g_{\mathrm{TGF}}^{2}$ & \multicolumn{1}{c|}{$g_{\mathrm{SF}}^{2}$} & $\mathrm{~L} / \mathrm{a}$ & $\beta$ & $g_{\mathrm{TGF}}^{2}$ & \multicolumn{1}{c|}{$g_{\mathrm{SF}}^{2}$} \\
\hline 8 & 40 & $0.167587(25)$ & $0.166813(18)$ & 12 & 40 & $0.169048(26)$ & $0.168350(19)$ \\
& 60 & $0.107511(18)$ & $0.107154(13)$ & & 60 & $0.108094(14)$ & $0.1077858(82)$ \\
& 80 & $0.079132(18)$ & $0.0789374(73)$ & & 80 & $0.079439(11)$ & $0.079294(10)$ \\
\hline 10 & 40 & $0.168404(22)$ & $0.167642(19)$ & 16 & 40 & $0.170093(21)$ & $0.169426(19)$ \\
& 60 & $0.107848(16)$ & $0.107478(15)$ & & 60 & $0.108526(19)$ & $0.108242(13)$ \\
& 80 & $0.079311(15)$ & $0.0791399(81)$ & & 80 & $0.079700(16)$ & $0.0795263(79)$ \\
\hline
\end{tabular}

Table 8. TGF and SF couplings on each lattice and each $\beta$ in the weak coupling region.

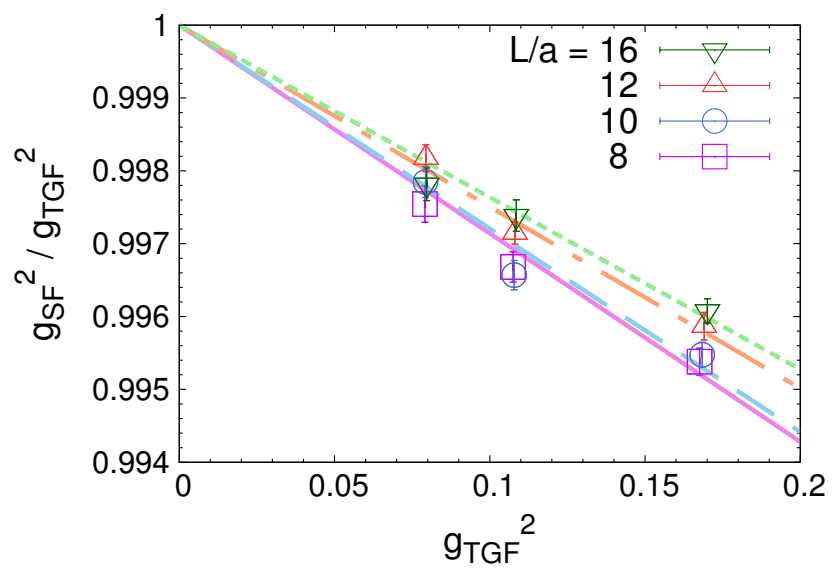

Figure 7. The ratio between the SF coupling and TGF coupling vs the TGF coupling. The lines show the fit results with linear fitting.

The ratio of the $\Lambda$-parameters is defined by

$$
\frac{\Lambda_{\mathrm{SF}}}{\Lambda_{\mathrm{TGF}}}=c \times \exp \left[\frac{c_{\mathrm{g}}^{(0)}}{2 b_{0}}\right] .
$$

with $c_{\mathrm{g}}^{(0)}$ from the continuum limit of $c_{\mathrm{g}}(a / L)$.

In table 8 we list the TGF and SF couplings measured on each lattice size and each $\beta$. Figure 7 shows $g_{\mathrm{SF}}^{2}(a / L, \beta) / g_{\mathrm{TGF}}^{2}(a / L, \beta)$ as a function of $g_{\mathrm{TGF}}^{2}(a / L, \beta)$. We fit the data linearly in $g_{\mathrm{TGF}}^{2}$ and the lines drawn in the figure are the fit results. Table 9 summarizes the fitted value of $c_{\mathrm{g}}(a / L)$ for each $L / a$. In figure 8, we plot $c_{\mathrm{g}}(a / L)$ as a function of $(a / L)^{2}$. Fitting the data linearly in $(a / L)^{2}$, we obtain

$$
c_{\mathrm{g}}^{(0)}=-0.02215(99)
$$

with $\chi^{2} / \mathrm{DoF} \simeq 1.48$. Consequently, eq. (6.4) with $c=0.3$ yields

$$
\frac{\Lambda_{\mathrm{SF}}}{c \Lambda_{\mathrm{TGF}}}=0.8530(61)
$$

where the error quoted is the statistical one. 


\begin{tabular}{|clc|ccc|}
\hline$L / a$ & $c_{\mathrm{g}}(L / a)$ & $\chi^{2} / \mathrm{DoF}$ & $L / a$ & $c_{\mathrm{g}}(L / a)$ & $\chi^{2} / \mathrm{DoF}$ \\
\hline 8 & $-0.02859(92)$ & 1.42 & 12 & $-0.02492(82)$ & 0.98 \\
10 & $-0.02793(85)$ & 2.76 & 16 & $-0.02363(84)$ & 1.11 \\
\hline
\end{tabular}

Table 9. The fit results for $c_{\mathrm{g}}$ at each lattice.

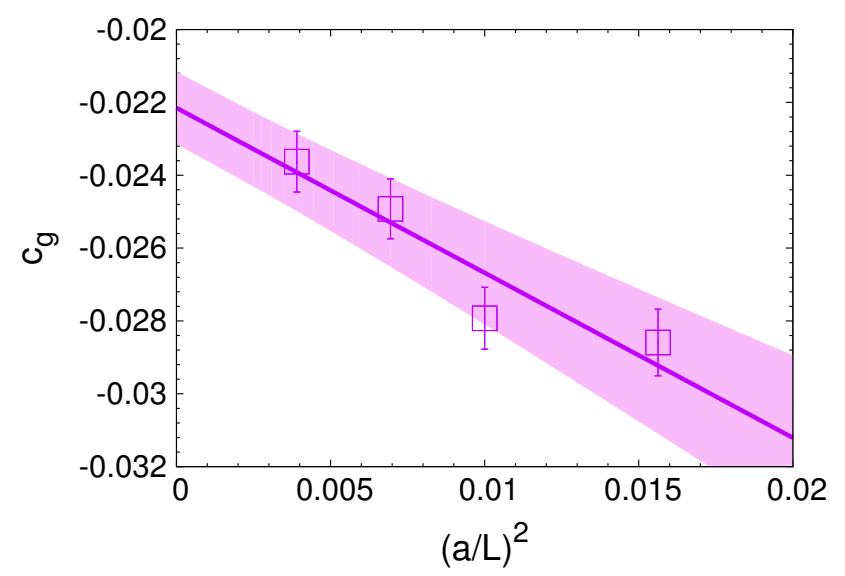

Figure 8. The coefficient $c_{\mathrm{g}}(a / L)$ vs $(a / L)^{2}$.

We can now evaluate $\Lambda_{\overline{\mathrm{MS}}}$ according to our strategy eq. (1.3). We assemble $\Lambda_{\mathrm{SF}} / \Lambda_{\overline{\mathrm{MS}}}=$ 0.48811(1) [12] and the results for $L_{\mathrm{max}} \Lambda_{\mathrm{TGF}}, L_{\mathrm{max}} A_{\mathrm{phys}}$, and $\Lambda_{\mathrm{SF}} / \Lambda_{\mathrm{TGF}}$ (tables 5,7 and eq. (6.6), respectively). Figures 9 and 10 show the renormalization condition $u^{*}$ dependence of $\Lambda_{\overline{\mathrm{MS}}} / \sqrt{\sigma}$ and $r_{0} \Lambda_{\overline{\mathrm{MS}}}$, respectively. All results with $N_{\max }=3$ and 4 from sets (B) and (C) are plotted. The dashed lines with gray band are from refs. [37] and [38] for comparison. We observe no renormalization condition dependence as expected. For the case of $N_{\max }=3$ the results are consistent between the sets (B) and (C). This also holds for the case of $N_{\max }=4$. On the other hand, the results between $N_{\max }=3$ and 4 are consistent within one standard deviation in the set (C), while the results between $N_{\max }=3$ and 4 differ by more than one standard deviation in the set (B). Our final estimates are

$$
\begin{aligned}
\frac{\Lambda_{\overline{\mathrm{MS}}}}{\sqrt{\sigma}} & =0.5315(81)_{\text {stat. }}\left({ }_{-48}^{+269}\right)_{\text {syst. }}, \\
r_{0} \Lambda_{\overline{\mathrm{MS}}} & =0.6062(92)_{\text {stat. }}\left({ }_{-52}^{+309}\right)_{\text {syst. }} .
\end{aligned}
$$

The central values are obtained by averaging the results with $N_{\max }=3$ from the set (C) over different renormalization conditions $u^{*}$. The upper and lower systematic errors are assigned by the discrepancies to the set (B) with $N_{\max }=4$ and to the set (B) with $N_{\max }=3$, respectively. Although there still remain large systematic errors coming from the form of the fitting function for the discrete beta function, our results of $\Lambda_{\overline{\mathrm{MS}}} / \sqrt{\sigma}$ and $r_{0} \Lambda_{\overline{\mathrm{MS}}}$ are consistent with the known values $\Lambda_{\overline{\mathrm{MS}}} / \sqrt{\sigma}=0.555\left(_{-17}^{+19}\right)$ from [37] and $r_{0} \Lambda_{\overline{\mathrm{MS}}}=0.62(2)$ from [38], respectively. 


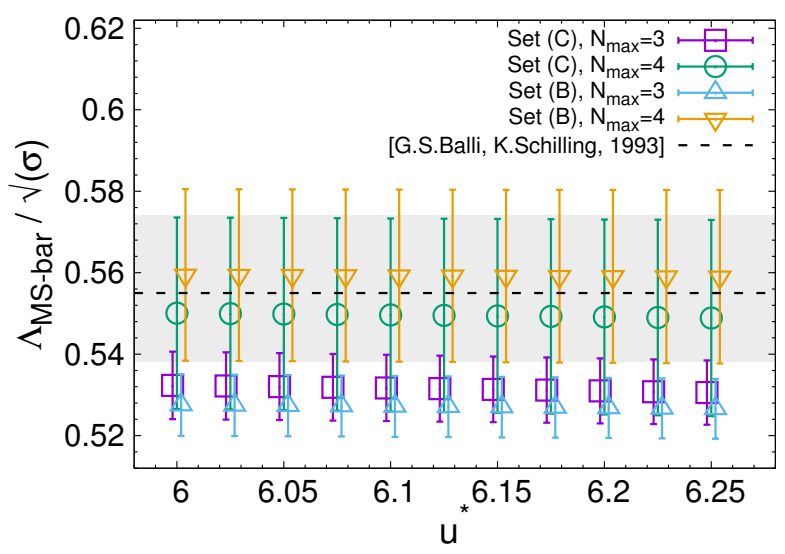

Figure 9. The intermediate scale $\left(u^{*}=g_{\mathrm{TGF}}^{2}\left(1 / L_{\mathrm{max}}\right)\right)$ dependence of our results $\Lambda_{\overline{\mathrm{MS}}} / \sqrt{\sigma}$. The dashed line is the known value $\Lambda_{\overline{\mathrm{MS}}} / \sqrt{\sigma}=0.555\left(_{-17}^{+19}\right)[37]$ and the gray band denotes $1 \sigma$. Symbols are slightly shifted horizontally for clarity.

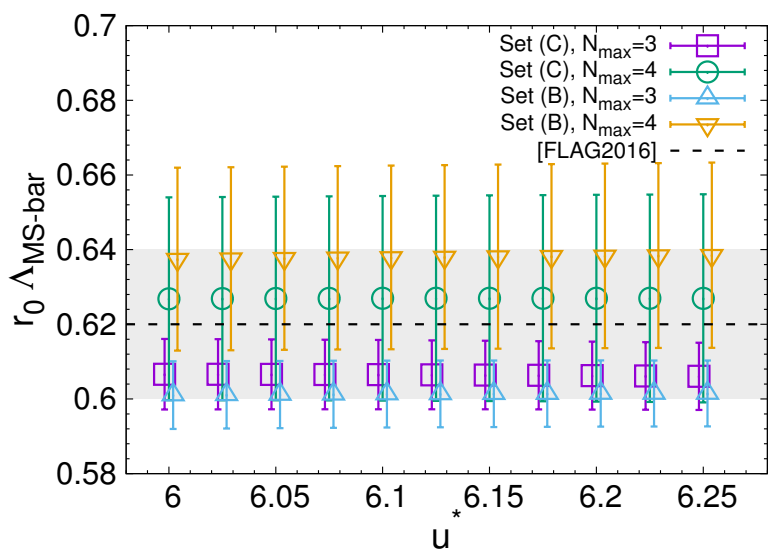

Figure 10. Same as figure 9, but for $r_{0} \Lambda_{\overline{\mathrm{MS}}}$. The dashed line with gray band is the known value $r_{0} \Lambda_{\overline{\mathrm{MS}}}=0.62(2)[38]$.

\section{Summary}

We have evaluated the $\Lambda$-parameter in the $\overline{\mathrm{MS}}$ scheme for the pure $\mathrm{SU}(3)$ gauge theory via the twisted gradient flow method according to our strategy shown in (1.3). Our results are summarized in eqs. (6.7) and (6.8), which are determined from two independent low energy physical scales, and both results are consistent with the known values in the literature. To obtain the results we have determined the $\Lambda$-parameter ratio between the TGF scheme and the SF scheme with lattice simulations, which is a non-trivial step in our analysis. Having obtained consistent values to the known ones in eqs. (6.7) and (6.8), we verified the ratio $\Lambda_{\mathrm{SF}} / \Lambda_{\mathrm{TGF}}$ (6.6) determined with non-perturbative simulations. To further confirm the value of the ratio $\Lambda_{\mathrm{SF}} / \Lambda_{\mathrm{TGF}}$, it would be interesting to compare our ratio with the analytic one from the explicit perturbative calculation [28].

The major source of the error of the $\Lambda$-parameter is the uncertainty of the fitting function for the discrete beta function, which we disentangled from the statistical error 
using high precision numerical computation. To further reduce the uncertainty, there are two possible strategies; first one is to increase the statistics in the strong coupling region, $u^{\max }=6.0-7.0$. However, since we observed a long auto-correlation time in this region, we have to treat this behavior carefully in the analysis. Another possibility is to increase the data point and the statistics in the region $u=5.0-6.0$, where no long auto-correlation time is observed. We expect stabilization of the fitting for the discrete beta function as a polynomial function of $u$ by lowering the maximum of the fit range with the increased data points and statistics in this weaker coupling region. Although the Sommer scale is available in $u^{*}=5.0-6.0$, the string tension is not available. Our prime interest was to demonstrate the strategy and to show the consistency, and we employed two independent low energy hadronic scales for the $\Lambda$-parameter. Therefore further study based on the second possibility is beyond the scope of this paper as it requires the string tension in the weak coupling region and more computational resources.

\section{Acknowledgments}

The numerical simulations have been done on the INSAM (Institute for Nonlinear Sciences and Applied Mathematics) cluster system at Hiroshima University, the Tatara system at the Research Institute for Information Technology of Kyushu University, and the HA-PACS system at the CCS (Center for Computational Sciences), University of Tsukuba. This work was partly supported by JSPS KAKENHI Grant Numbers 17K05417 and 16K05326. I. K. is supported by MEXT as "Priority Issue on Post-K computer" (Elucidation of the Fundamental Laws and Evolution of the Universe) and JICFuS, by which K.-I. I. is also partially supported.

\section{A Derivation of the action}

In this appendix, we derive the action with periodic variables (2.1). We start from the following action in $\mathrm{SU}\left(N_{\mathrm{C}}\right)$ defined on a $(L / a)^{4} \equiv \hat{L}^{4}$ lattice with the twisted boundary condition on the $x-y$ plane and periodic boundary condition in $z$ and $t$ directions:

$$
S=\frac{\beta}{2 N_{\mathrm{C}}} \sum_{\substack{n, \mu, \nu \\ \mu \neq \nu)}} \operatorname{Tr}\left[P_{\mu \nu}[n ; V]\right],
$$

where

$$
P_{\mu \nu}[n ; V]=V_{\mu}(n) V_{\nu}(n+\hat{\mu}) V_{\mu}^{\dagger}(n+\hat{\nu}) V_{\nu}^{\dagger}(n)
$$

is a plaquette variable made of link variables $V_{\mu}(n)$ with the twisted boundary condition:

$$
\begin{array}{ll}
V_{\mu}(n+\hat{L} \hat{\nu})=\Gamma_{\nu} V_{\mu}(n) \Gamma_{\nu}^{\dagger} & (\nu=1,2), \\
V_{\mu}(n+\hat{L} \hat{\nu})=V_{\mu}(n) & (\nu=3,4),
\end{array}
$$

where $N_{\mathrm{C}} \times N_{\mathrm{C}}$ unitary matrix $\Gamma_{\nu}(\nu=1,2)$ is called twist matrix and satisfies

$$
\Gamma_{1} \Gamma_{2}=\omega \Gamma_{2} \Gamma_{1}, \quad \omega=\exp \left[\frac{2 \pi i}{N_{\mathrm{C}}}\right] .
$$


Let us eliminate the link variables on $n_{1}=0$ or $n_{2}=0$ by using the variables on $n_{i}=\hat{L}$. The plaquette on $n_{1}=n_{2}=0$ becomes

$$
\begin{aligned}
\operatorname{Tr} P_{12}\left[\left(0,0, n_{3}, n_{4}\right) ; V\right]=\omega^{*} \operatorname{Tr}[ & V_{1}\left(\hat{L}, \hat{L}, n_{3}, n_{4}\right) \Gamma_{1} V_{2}\left(1, \hat{L}, n_{3}, n_{4}\right) \Gamma_{2} \\
& \left.\Gamma_{1}^{\dagger} V_{1}^{\dagger}\left(\hat{L}, 1, n_{3}, n_{4}\right) \Gamma_{2}^{\dagger} V_{2}^{\dagger}\left(\hat{L}, \hat{L}, n_{3}, n_{4}\right)\right] .
\end{aligned}
$$

By introducing the following new variables for $n_{1,2}=1,2, \ldots, \hat{L}$

$$
\begin{aligned}
U_{1}\left(\hat{L}, n_{2}, n_{3}, n_{4}\right) & \equiv V_{1}\left(\hat{L}, n_{2}, n_{3}, n_{4}\right) \Gamma_{1}=\Gamma_{1} V_{1}\left(0, n_{2}, n_{3}, n_{4}\right), \\
U_{2}\left(n_{1}, \hat{L}, n_{3}, n_{4}\right) & \equiv V_{2}\left(n_{1}, \hat{L}, n_{3}, n_{4}\right) \Gamma_{2}=\Gamma_{2} V_{2}\left(n_{1}, 0, n_{3}, n_{4}\right), \\
U_{\mu}\left(n_{1}, n_{2}, n_{3}, n_{4}\right) & \equiv V_{\mu}\left(n_{1}, n_{2}, n_{3}, n_{4}\right) \quad \text { for others, }
\end{aligned}
$$

it becomes

$$
\begin{array}{r}
\operatorname{Tr} P_{12}\left[\left(0,0, n_{3}, n_{4}\right) ; V\right]=\omega^{*} \operatorname{Tr}[ \\
U_{1}\left(\hat{L}, \hat{L}, n_{3}, n_{4}\right) U_{2}\left(1, \hat{L}, n_{3}, n_{4}\right) \\
\left.U_{1}^{\dagger}\left(\hat{L}, 1, n_{3}, n_{4}\right) U_{2}^{\dagger}\left(\hat{L}, \hat{L}, n_{3}, n_{4}\right)\right] .
\end{array}
$$

Except for the overall factor $\omega$, this is exactly the plaquette with periodic link variables $U_{\mu}(n)$. Therefore we define link variables on $n_{1}=0$ and $n_{2}=0$ through the periodic boundary condition:

$$
\begin{aligned}
U_{\mu}\left(0, n_{2}, n_{3}, n_{4}\right) & \equiv U_{\mu}\left(\hat{L}, n_{2}, n_{3}, n_{4}\right), \\
U_{\mu}\left(n_{1}, 0, n_{3}, n_{4}\right) & \equiv U_{\mu}\left(n_{1}, \hat{L}, n_{3}, n_{4}\right), \\
U_{\mu}\left(0,0, n_{3}, n_{4}\right) & \equiv U_{\mu}\left(\hat{L}, \hat{L}, n_{3}, n_{4}\right) .
\end{aligned}
$$

Similar calculations show other plaquettes become those with $U_{\mu}(n)$ without overall factor. Then, we finally obtain the action with periodic link variable $U_{\mu}(n)$

$$
S=\frac{\beta}{2 N_{\mathrm{C}}} \sum_{\substack{n, \mu, \nu \\(\mu \neq \nu)}} \operatorname{Tr}\left[Z_{\mu \nu}(n) P_{\mu \nu}[n ; U]\right],
$$

where $Z_{\mu \nu}(n)=Z_{\nu \mu}^{*}(n)$ is given as

$$
Z_{\mu \nu}(n)= \begin{cases}\omega^{*} & \mu=1, \nu=2, \text { and } n_{1}=n_{2}=0 \\ 1 & \text { otherwise }\end{cases}
$$

\section{B The number of the configurations for $g_{\text {TGF }}^{2}$}

We list the number of the configurations used to calculate $g_{\mathrm{TGF}}^{2}$ in table 10 . 


\begin{tabular}{|c|c|c|c|c|c|c|}
\hline \multirow[b]{2}{*}{$\beta$} & \multicolumn{6}{|c|}{ Number of configurations $\left[\tau_{\text {int }}\right]$} \\
\hline & 12 & 16 & 18 & 24 & 36 & $L / a$ \\
\hline 6.11 & $91300[1.7]$ & & & & & \\
\hline 6.13 & $89600[1.7]$ & & & & & \\
\hline 6.15 & $89600[1.8]$ & & & & & \\
\hline 6.17 & $89600[1.9]$ & & & & & \\
\hline 6.20 & $69500[2.3]$ & & & & & \\
\hline 6.25 & $37600[1.9]$ & & & & & \\
\hline 6.30 & & $27500[3.5]$ & & & & \\
\hline 6.31 & & $29600[4.2]$ & & & & \\
\hline 6.33 & $37100[1.9]$ & $29600[4.6]$ & & & & \\
\hline 6.36 & & $29600[4.7]$ & & & & \\
\hline 6.38 & & $29100[6.9]$ & $16760[5.3]$ & & & \\
\hline 6.40 & $19500[1.0]$ & $26000[8.8]$ & $15266[4.9]$ & & & \\
\hline 6.42 & $19600[0.9]$ & $29100[4.5]$ & $27620[7.1]$ & & & \\
\hline 6.45 & $19600[1.1]$ & $29100[6.0]$ & $25820[7.2]$ & & & \\
\hline 6.47 & & & $18020[11.3]$ & & & \\
\hline 6.49 & & & $18020[8.6]$ & & & \\
\hline 6.50 & $15500[0.8]$ & $14300[8.7]$ & $14804[7.8]$ & & & \\
\hline 6.60 & $19600[0.6]$ & $14350[1.3]$ & $10020[7.2]$ & $9340[10.5]$ & & \\
\hline 6.62 & & $8550[0.9]$ & & $18240[15.0]$ & & \\
\hline 6.65 & $19600[0.7]$ & $9600[1.9]$ & $10020[9.9]$ & $8820[11.1]$ & & \\
\hline 6.75 & $15500[0.7]$ & $2500[1.0]$ & $2200[1.2]$ & $14950[3.8]$ & & \\
\hline 6.80 & & $8550[0.8]$ & & $9470[3.1]$ & & \\
\hline 6.86 & $19600[0.7]$ & $8050[0.9]$ & & $9420[2.3]$ & & \\
\hline 6.90 & & & & $163262[204]$ & $26610[14.9]$ & \\
\hline 6.94 & & & & $7440[2.0]$ & $41435[50.1]$ & \\
\hline 6.97 & & & & $8040[1.7]$ & $39240[65.3]$ & \\
\hline 7.00 & $15500[0.6]$ & $1600[1.3]$ & $1420[0.9]$ & $11006[1.6]$ & $20057[9.9]$ & \\
\hline 7.05 & & $9500[0.8]$ & & $10440[1.6]$ & & \\
\hline 7.13 & & & & $12710[1.8]$ & $20890[7.2]$ & \\
\hline 7.18 & & & & $12710[1.5]$ & $20850[4.7]$ & \\
\hline 7.25 & $15500[0.6]$ & $1750[0.6]$ & $1900[1.0]$ & $10650[1.3]$ & $14500[3.3]$ & \\
\hline 7.40 & & & & $10440[1.3]$ & $12145[3.3]$ & \\
\hline 7.50 & $15500[0.7]$ & $1300[0.6]$ & $1350[1.0]$ & $9570[1.3]$ & $12900[3.5]$ & \\
\hline 7.75 & $15500[0.7]$ & $1800[0.7]$ & $1200[0.9]$ & $9450[1.4]$ & $14500[2.7]$ & \\
\hline 8.00 & $15500[0.7]$ & $1100[0.7]$ & $1200[0.9]$ & $9450[1.5]$ & $13700[2.3]$ & \\
\hline 8.25 & $15000[0.7]$ & $1700[0.9]$ & $1480[0.9]$ & $9350[1.2]$ & $12900[2.8]$ & \\
\hline 8.50 & $15000[0.6]$ & $1400[0.7]$ & $1300[1.0]$ & $23620[1.4]$ & $19980[2.7]$ & \\
\hline 9.00 & $15500[0.7]$ & $1300[0.8]$ & $1100[1.5]$ & $22220[1.3]$ & $19920[2.8]$ & \\
\hline 9.50 & $15500[0.9]$ & $1800[1.1]$ & $1200[1.2]$ & $22400[1.6]$ & $19640[2.3]$ & \\
\hline 10.00 & $15500[0.7]$ & $1800[0.7]$ & $1600[0.7]$ & $23000[1.4]$ & $19640[3.2]$ & \\
\hline
\end{tabular}

Table 10. Number of configurations after thermalization used to calculate $g_{\mathrm{TGF}}^{2} \cdot \tau_{\text {int. }}$ indicates integrated autocorrelation. 


\section{Tables to evaluate $L_{\max } / A_{\text {phys }}$}

In tables 11, 12 and 13 we collect values needed to evaluate $L_{\max } / A_{\text {phys }}$ in section 5 .

\begin{tabular}{|c|clll|}
\hline & \multicolumn{3}{|c|}{$\beta^{*}$} & \\
$u^{*}$ & 12 & 16 & $L_{\max } / a^{*}$ \\
\hline 6.000 & $6.18803(21)$ & $6.38835(46)$ & $6.47494(71)$ & \\
6.025 & $6.18565(20)$ & $6.38578(46)$ & $6.47232(70)$ & \\
6.050 & $6.18330(20)$ & $6.38324(46)$ & $6.46974(69)$ & \\
6.075 & $6.18097(20)$ & $6.38073(45)$ & $6.46718(68)$ & \\
6.100 & $6.17866(20)$ & $6.37824(45)$ & $6.46464(67)$ & \\
6.125 & $6.17638(20)$ & $6.37577(45)$ & $6.46213(66)$ & \\
6.150 & $6.17411(19)$ & $6.37333(44)$ & $6.45964(65)$ & \\
6.175 & $6.17187(19)$ & $6.37091(44)$ & $6.45718(64)$ & \\
6.200 & $6.16965(19)$ & $6.36851(43)$ & $6.45474(63)$ & \\
6.225 & $6.16745(19)$ & $6.36614(43)$ & $6.45233(62)$ & \\
6.250 & $6.16528(18)$ & $6.36378(42)$ & $6.44994(61)$ & \\
\hline
\end{tabular}

Table 11. The bare coupling $\beta^{*}$ at the renormalization condition $u^{*}$.

\begin{tabular}{|c|cccc|}
\hline & \multicolumn{4}{|c|}{$\left(L_{\max } / a^{*}\right) \cdot\left(a^{*} \sqrt{\sigma}\right)$} \\
$u^{*}$ & 12 & 16 & 18 & $L_{\max } / a^{*}$ \\
\hline 6.000 & $1.9207(36)$ & $1.9228(39)$ & $1.9156(57)$ & \\
6.025 & $1.9275(36)$ & $1.9297(40)$ & $1.9227(56)$ & \\
6.050 & $1.9343(36)$ & $1.9366(40)$ & $1.9297(56)$ & \\
6.075 & $1.9410(36)$ & $1.9434(40)$ & $1.9367(55)$ & \\
6.100 & $1.9477(36)$ & $1.9502(40)$ & $1.9436(54)$ & \\
6.125 & $1.9544(36)$ & $1.9570(40)$ & $1.9505(54)$ & \\
6.150 & $1.9610(36)$ & $1.9637(40)$ & $1.9573(53)$ \\
6.175 & $1.9676(36)$ & $1.9704(40)$ & $1.9641(52)$ & \\
6.200 & $1.9742(36)$ & $1.9770(40)$ & $1.9708(52)$ \\
6.225 & $1.9807(36)$ & $1.9836(41)$ & $1.9776(51)$ \\
6.250 & $1.9872(36)$ & $1.9902(41)$ & $1.9842(51)$ & \\
\hline
\end{tabular}

Table 12. $L_{\max } \sqrt{\sigma}$ at each $u^{*}$. 


\begin{tabular}{|c|cccc|}
\hline & \multicolumn{4}{|c|}{$\left(L_{\max } / a^{*}\right) \cdot\left(a^{*} / r_{0}\right)$} \\
$u^{*}$ & 12 & 16 & 18 & $L_{\max } / a^{*}$ \\
\hline 6.000 & $1.6592(38)$ & $1.6697(51)$ & $1.6732(61)$ & \\
6.025 & $1.6651(38)$ & $1.6755(51)$ & $1.6790(61)$ & \\
6.050 & $1.6709(38)$ & $1.6813(51)$ & $1.6848(61)$ & \\
6.075 & $1.6766(38)$ & $1.6870(51)$ & $1.6905(61)$ & \\
6.100 & $1.6824(38)$ & $1.6927(51)$ & $1.6962(60)$ & \\
6.125 & $1.6881(38)$ & $1.6984(51)$ & $1.7019(60)$ \\
6.150 & $1.6938(38)$ & $1.7040(50)$ & $1.7075(60)$ \\
6.175 & $1.6995(38)$ & $1.7096(50)$ & $1.7131(60)$ & \\
6.200 & $1.7051(38)$ & $1.7152(50)$ & $1.7186(60)$ \\
6.225 & $1.7107(38)$ & $1.7207(50)$ & $1.7242(60)$ \\
6.250 & $1.7163(38)$ & $1.7262(50)$ & $1.7297(60)$ & \\
\hline
\end{tabular}

Table 13. $L_{\max } / r_{0}$ at each $u^{*}$.

Open Access. This article is distributed under the terms of the Creative Commons Attribution License (CC-BY 4.0), which permits any use, distribution and reproduction in any medium, provided the original author(s) and source are credited.

\section{References}

[1] C. Allton, M. Teper and A. Trivini, On the running of the bare coupling in $\mathrm{SU}(N)$ lattice gauge theories, JHEP 07 (2008) 021 [arXiv:0803.1092] [INSPIRE].

[2] G. Parisi, Recent progresses in gauge theories, World Sci. Lect. Notes Phys. 49 (1980) 349 [AIP Conf. Proc. 68 (1980) 1531] [LNF-80-52-P] [INSPIRE].

[3] M. Lüscher, P. Weisz and U. Wolff, A numerical method to compute the running coupling in asymptotically free theories, Nucl. Phys. B 359 (1991) 221 [InSPIRE].

[4] M. Lüscher, R. Narayanan, P. Weisz and U. Wolff, The Schrödinger functional: a renormalizable probe for non-Abelian gauge theories, Nucl. Phys. B 384 (1992) 168 [hep-lat/9207009] [INSPIRE].

[5] M. Lüscher, R. Sommer, U. Wolff and P. Weisz, Computation of the running coupling in the SU(2) Yang-Mills theory, Nucl. Phys. B 389 (1993) 247 [hep-lat/9207010] [InSPIRE].

[6] M. Lüscher, R. Sommer, P. Weisz and U. Wolff, A precise determination of the running coupling in the SU(3) Yang-Mills theory, Nucl. Phys. B 413 (1994) 481 [hep-lat/9309005] [INSPIRE].

[7] ALPHA collaboration, A. Bode, P. Weisz and U. Wolff, Two loop computation of the Schrödinger functional in lattice QCD, Nucl. Phys. B 576 (2000) 517 [Erratum ibid. B 600 (2001) 453] [Erratum ibid. B 608 (2001) 481] [hep-lat/9911018] [INSPIRE]. 
[8] ALPHA collaboration, M. Della Morte, R. Frezzotti, J. Heitger, J. Rolf, R. Sommer and U. Wolff, Computation of the strong coupling in QCD with two dynamical flavors, Nucl. Phys. B 713 (2005) 378 [hep-lat/0411025] [INSPIRE].

[9] ALPHA collaboration, F. Tekin, R. Sommer and U. Wolff, The running coupling of QCD with four flavors, Nucl. Phys. B 840 (2010) 114 [arXiv: 1006.0672] [INSPIRE].

[10] S. Capitani, M. Lüscher, R. Sommer and H. Wittig, Non-perturbative quark mass renormalization in quenched lattice QCD, Nucl. Phys. B 544 (1999) 669 [Erratum ibid. B 582 (2000) 762] [hep-lat/9810063] [INSPIRE].

[11] M. Dalla Brida and M. Lüscher, SMD-based numerical stochastic perturbation theory, Eur. Phys. J. C 77 (2017) 308 [arXiv: 1703.04396] [INSPIRE].

[12] S. Sint and R. Sommer, The running coupling from the QCD Schrödinger functional: a one loop analysis, Nucl. Phys. B 465 (1996) 71 [hep-lat/9508012] [INSPIRE].

[13] G.M. de Divitiis, R. Frezzotti, M. Guagnelli and R. Petronzio, A definition of the running coupling constant in a twisted SU(2) lattice gauge theory, Nucl. Phys. B 422 (1994) 382 [hep-lat/9312085] [INSPIRE].

[14] G.M. de Divitiis, R. Frezzotti, M. Guagnelli and R. Petronzio, Nonperturbative determination of the running coupling constant in quenched $\mathrm{SU}(2)$, Nucl. Phys. B 433 (1995) 390 [hep-lat/9407028] [INSPIRE].

[15] Alpha collaboration, G. de Divitiis et al., Universality and the approach to the continuum limit in lattice gauge theory, Nucl. Phys. B 437 (1995) 447 [hep-lat/9411017] [INSPIRE].

[16] E. Bilgici et al., A new scheme for the running coupling constant in gauge theories using Wilson loops, Phys. Rev. D 80 (2009) 034507 [arXiv: 0902.3768] [INSPIRE].

[17] E. Itou, Properties of the twisted Polyakov loop coupling and the infrared fixed point in the SU(3) gauge theories, PTEP 2013 (2013) 083B01 [arXiv:1212.1353] [INSPIRE].

[18] R. Narayanan and H. Neuberger, Infinite $N$ phase transitions in continuum Wilson loop operators, JHEP 03 (2006) 064 [hep-th/0601210] [INSPIRE].

[19] M. Lüscher, Properties and uses of the Wilson flow in lattice QCD, JHEP 08 (2010) 071 [Erratum ibid. 03 (2014) 092] [arXiv: 1006 .4518] [INSPIRE].

[20] M. Lüscher and P. Weisz, Perturbative analysis of the gradient flow in non-Abelian gauge theories, JHEP 02 (2011) 051 [arXiv:1101.0963] [INSPIRE].

[21] Z. Fodor, K. Holland, J. Kuti, D. Nogradi and C.H. Wong, The Yang-Mills gradient flow in finite volume, JHEP 11 (2012) 007 [arXiv:1208.1051] [INSPIRE].

[22] A. Ramos, The gradient flow running coupling with twisted boundary conditions, JHEP 11 (2014) 101 [arXiv: 1409.1445] [INSPIRE].

[23] V. Leino, J. Rantaharju, T. Rantalaiho, K. Rummukainen, J.M. Suorsa and K. Tuominen, The gradient flow running coupling in $\mathrm{SU}(2)$ gauge theory with $N_{f}=8$ fundamental flavors, Phys. Rev. D 95 (2017) 114516 [arXiv:1701.04666] [INSPIRE].

[24] C.-J.D. Lin, K. Ogawa and A. Ramos, The Yang-Mills gradient flow and SU(3) gauge theory with 12 massless fundamental fermions in a colour-twisted box, JHEP 12 (2015) 103 [arXiv: 1510.05755] [INSPIRE].

[25] P. Fritzsch and A. Ramos, The gradient flow coupling in the Schrödinger functional, JHEP 10 (2013) 008 [arXiv: 1301.4388] [INSPIRE]. 
[26] ALPHA collaboration, M. Dalla Brida, P. Fritzsch, T. Korzec, A. Ramos, S. Sint and R. Sommer, Slow running of the gradient flow coupling from $200 \mathrm{MeV}$ to $4 \mathrm{GeV}$ in $N_{f}=3$ QCD, Phys. Rev. D 95 (2017) 014507 [arXiv:1607.06423] [INSPIRE].

[27] ALPHA collaboration, M. Dalla Brida, P. Fritzsch, T. Korzec, A. Ramos, S. Sint and R. Sommer, A status update on the determination of $\Lambda_{\mathrm{MS}}^{N_{f}=3}$ by the ALPHA collaboration, PoS (LATTICE 2015) 248 [arXiv: 1511.05831] [INSPIRE].

[28] E.I. Bribian and M. Garcia Perez, Perturbative running of the twisted Yang-Mills coupling in the gradient flow scheme, PoS (LATTICE2016) 371 [arXiv:1611.07221] [INSPIRE].

[29] M. Asakawa, T. Hatsuda, T. Iritani, E. Itou, M. Kitazawa and H. Suzuki, Determination of reference scales for Wilson gauge action from Yang-Mills gradient flow, arXiv:1503.06516 [INSPIRE].

[30] A. González-Arroyo and M. Okawa, The string tension from smeared Wilson loops at large-N, Phys. Lett. B 718 (2013) 1524 [arXiv:1206.0049] [INSPIRE].

[31] S. Necco and R. Sommer, The $N_{f}=0$ heavy quark potential from short to intermediate distances, Nucl. Phys. B 622 (2002) 328 [hep-lat/0108008] [INSPIRE].

[32] K.-I. Ishikawa, I. Kanamori, Y. Murakami, A. Nakamura, M. Okawa and R. Ueno, Numerical determination of the $\Lambda$-parameter in $\mathrm{SU}(3)$ gauge theory from the twisted gradient flow coupling, PoS (LATTICE2016) 185 [arXiv: 1612.01676] [INSPIRE].

[33] K. Fabricius and O. Haan, Heat bath method for the twisted Eguchi-Kawai model, Phys. Lett. B 143 (1984) 459 [INSPIRE].

[34] ALPHA collaboration, U. Wolff, Monte Carlo errors with less errors, Comput. Phys. Commun. 156 (2004) 143 [Erratum ibid. 176 (2007) 383] [hep-lat/0306017] [INSPIRE].

[35] Alpha collaboration, A. Bode, U. Wolff and P. Weisz, Two loop computation of the Schrödinger functional in pure SU(3) lattice gauge theory, Nucl. Phys. B 540 (1999) 491 [hep-lat/9809175] [INSPIRE].

[36] T. Awaya, Two-dimensional curve fitting in counting experiments, Nucl. Instrum. Meth. 212 (1983) 311.

[37] G.S. Bali and K. Schilling, Running coupling and the $\Lambda$ parameter from $\mathrm{SU}(3)$ lattice simulations, Phys. Rev. D 47 (1993) 661 [hep-lat/9208028] [INSPIRE].

[38] S. Aoki et al., Review of lattice results concerning low-energy particle physics, Eur. Phys. J. C 77 (2017) 112 [arXiv: 1607.00299] [INSPIRE]. 